Journal of Applied AnALysis

Vol. 9, No. 1 (2003), pp. 75-102

\title{
MINIMAX SOLUTIONS OF THE DUAL HAMILTON-JACOBI EQUATION
}

\author{
A. NOWAKOWSKI \\ Received June 7, 2001 and, in revised form, January 23, 2003
}

\begin{abstract}
In the paper the existence and continuous dependence of a kind of minimax solution to the dual Hamilton-Jacobi equations is proved. The main difficulties which appear here are a special type of the boundary conditions and the transversality conditions which that solution must satisfy. That type of problems come from optimal control and game theory.
\end{abstract}

\section{Introduction}

Theories of fields of extremals and dynamic programming are fundamental methods in investigating sufficient optimality conditions for nonlinear classical and modern, variational and control problems. In papers [7] and [8] the dual theory of field of extremals and dual dynamic programming method are described. Both methods require new tools, new equations to the study. One of them is a dual dynamic Hamilton-Jacobi equation with a specific type of boundary condition (see [8]). We recall the main ideas of that method. To this effect let us start with the classical approach to dynamic programminig and denote by $T X \subset \mathbb{R}^{n+1}$ a set covered by graphs

2000 Mathematics Subject Classification. 35F25, 35F30, 35D05.

Key words and phrases. Dual Hamilton-Jacobi equation, minimax solutions, generalized solutions, generalized problem of Bolza.

ISSN 1425-6908（C) Heldermann Verlag. 
of trajectories of the field of extremals - concourse of flights. If one considers the optimal control problem of Bolza

$$
\operatorname{minimize} J(x, u)=\int_{0}^{T} L(t, x(t), u(t)) d t+l(x(T))
$$

subject to

$$
\begin{aligned}
\dot{x}(t)= & f(t, x(t), u(t)) \quad \text { in }[0, T], \\
& u(t) \in U \subset \mathbb{R}^{m}, \\
& x(0)=c, \quad c \in \mathbb{R}^{n},
\end{aligned}
$$

where $L:[0, T] \times \mathbb{R}^{n} \times \mathbb{R}^{m} \rightarrow \mathbb{R}, f:[0, T] \times \mathbb{R}^{n} \times \mathbb{R}^{m} \rightarrow \mathbb{R}^{n}$ and $l: \mathbb{R}^{n} \rightarrow \mathbb{R}$ then one defines the value function as

$$
S(t, x)=\inf \left\{\int_{t}^{T} L(\tau, x(\tau), u(\tau)) d t+l(x(T))\right\}
$$

where the infimum is taken over admissible pairs $x(\tau), u(\tau), \tau \in[t, T]$, whose trajectories start at $(t, x) \in T X$ and graphs are contained in $T X$. By [7] the existence of value function (4) is determined by the existence of a concourse of flights (field of extremals). Futher it was concluded that if $S(t, x)$ is differentiable then it satisfies the partial differential equation

$$
S_{t}(t, x)+H\left(t, x, S_{x}(t, x)\right)=0,
$$

where $H(t, x, y)=y f(t, x, u(t, x))+L(t, x, u(t, x))$ and $u(t, x)$ is an optimal feedback control, and the partial differential equation of dynamic programming

$$
\min \left\{S_{t}(t, x)+S_{x}(t, x) f(t, x, u)+L(t, x, u): u \in U\right\}=0 .
$$

It is clear from that description of the value function that even if we make strong assumptions on the data of the problem (1)-(3) we can not expect that generaly the value function appears to be continuous. This is why in eighties and nineties have appeared many generalizations of solutions to (5) or (6). The most general are viscosity solutions to (5) or (6) (see e.g. [4], [10], [1], [3]). However even in that case the value function has to be at least lower semicontinuous [1], [3] (under very strong assumptions on the data). In many practical control problems these generalizations seem to be enough, but if we want to consider the above problem depended, additionaly, on second parameters (control) and consider "minmax" type problems (which appear e.g. in game theory see also [10], [6] and [9]) then requiring that the value function is at least lower semicontinuous may turn out to be to strong. Thus, in our opinion, we need still to search for new methods to study (4). In [7] the author suggested the second nonclassical approach to dynamic programming; the domain of exploration was carried out from the $(t, x)$ - space to the space of multipliers $\left(\left(t, y^{0}, y\right)\right.$-space $)$. 
Then another function was defined - the dual value function $-S_{D}(t, p)$ in a set $P \subset \mathbb{R}^{n+2}$ of the dual space $\left(t, y^{0}, y\right)=(t, p), y^{0} \leq 0$,

$$
S_{D}(t, p)=\inf \left\{-y^{0} \int_{t}^{T} L(\tau, x(\tau), u(\tau)) d t-y^{0} l(x(T))\right\}
$$

where the inferior is taken over pairs $(x(\tau), u(\tau)), \tau \in[t, T]$ satisfying (2), (3), whose trajectories start at $(t, x(t, p))(x(t, p)$ will be defined below) and their graphs are contained $T X$. By [7] the existence of $S_{d}(t, p)$ is determined by the existence of a concourse of flights. Next a new function was defined:

$$
V(t, p)=-S_{D}(t, p)-x(t, p) y=V_{y^{0}}(t, p) y^{0}+V_{y}(t, p) y,
$$

$\left(-S_{D}(t, p)=V_{y^{0}}(t, p) y^{0},-x(t, p)=V_{y}(t, p)\right),(t, p) \in(0, T) \times \mathbb{R}^{-} \times \mathbb{R}^{n}$, which satisfies the partial differential equation

$$
V_{t}(t, p)+H\left(t,-V_{y}(t, p), p\right)=0,(t, p)=\left(t, y^{0}, y\right) \in(0, T) \times \mathbb{R}^{-} \times \mathbb{R}^{n},
$$

where $H(t, v, p)=y^{0} L(t, v, u(t, p))+y f(t, v, u(t, p))$ and $u(t, p)$ is a dual optimal feedback control, $\mathbb{R}^{-}=\left\{y^{0} \in \mathbb{R}: y^{0} \leq 0\right\}$, and the dual partial differential equation programming

$$
\max \left\{V_{t}(t, p)+y f\left(t,-V_{y}(t, p), u\right)+y^{0} L\left(t,-V_{y}(t, p), u\right): u \in U\right\} .
$$

We require that the function $V$ satisfies a special type of the boundary condition (see [8, Theorem 3.1])

$$
y^{0} V_{y^{0}}(T, p)=y^{0} l\left(-V_{y}(T, p)\right) .
$$

Conversely, if a function $V$ satisfies $(9),(8),(10)$ then $S_{D}(t, p)$, calculated from (7), is a dual value function for problem (1), (2), (3) (see [8]). Moreover the original value function satisfies the relation: $-y^{0} S(t, x(t, p))=$ $-y^{0} V_{y^{0}}(t, p)$ with $x(t, p)=-V_{y}(t, p),(t, p) \in P$. It is clear that even if $V(t, p)$ is of $C^{1}$ then $S(t, x)$ need not be continuous.

The above dual problem can be solved in the form described only if we are looking for classical solutions i.e. solutions being functions of $C^{1}$ (having continuous partial derivatives). However the existence of classical solutions require very strong assumptions on the Hamiltonian $H$, which, in applications, e.g. optimal control theory or differential games are almost never satisfied. That is why we need a new type of solutions, namely generalized solutions. In the literature one meets many generalized solutions of the classical Cauchy initial problem for the Hamilton-Jacobi equation (see e.g. [1], [2], [3], [10], [4], [5]). Our construction of a generalized solution a kind of minimax solution - for the dual problem and the proof of the existence of solution are based, in its idea, on the book of [10], where the primal problem is considered and the construction of the minimax solutions for (5) are given. 
The aim of the paper is to prove the existence and continuous dependence of a kind of generalized solution for dual problem (8)-(10). We will require that $V(t, p)$ is continuous only. Thus the original value function need not be even lower semicontinuous. Moreover we impose different assumption on our Hamiltonian than it is usually done (see (H1)-(H4) and [10], [1], [3]).

\section{The definition of generalized solutions}

In order to formulate a generalized solution we need several notions and notations. Let

$$
X c=\left\{x \in \mathbb{R}^{n}:|x|=1\right\}, \quad B=\left\{x \in \mathbb{R}^{n}:|x| \leq 1\right\} .
$$

Let us consider Hamiltonian $H:[0, T] \times \mathbb{R}^{n} \times \mathbb{R}^{n+1} \rightarrow \mathbb{R}$ of the variables $(t, x, p)$ satisfying the following hypotheses:

H1 For each $x \in \mathbb{R}^{n}$ the function $(t, p) \rightarrow H(t, x, p)$ is continuous in $G=(0, T) \times \mathbb{R}^{n+1}$.

H2 For each bounded set $E \subset G$, there exists a number $\Lambda>0$ such that, for all $\left(t, p^{\prime}\right) \in E,\left(t, p^{\prime \prime}\right) \in E, x \in X c$ the following inequality holds:

$$
\left|H\left(t, x, p^{\prime}\right)-H\left(t, x, p^{\prime \prime}\right)\right| \leq \Lambda\left|p^{\prime}-p^{\prime \prime}\right| .
$$

H3 For each $(t, p) \in G$, the Lipschitz condition with respect to $x$ is satisfied:

$$
\sup _{x^{\prime} \in B, x^{\prime \prime} \in B}\left[\left|H\left(t, x^{\prime}, p\right)-H\left(t, x^{\prime \prime}, p\right)\right|-L(t, p)\left|x^{\prime}-x^{\prime \prime}\right|\right] \leq 0,
$$

where $(t, p) \rightarrow L(t, p)$ is continuous in $G$ and subject to the following conditions with some constant $k>0$ :

$$
L(t, p) \leq k(1+|p|),(t, p) \in G .
$$

H4 For each $(t, p) \in G$, the function $x \rightarrow H(t, x, p)$ is positively homogeneous, i.e.

$$
H(t, a x, p)=a H(t, x, p), \text { for } a>0 .
$$

The hypothesis (H4) will get rid of in Section 4, but the first construction of generalized solution is more convenient to be carried out with this assumption. Let us observe that hypotheses $(\mathbf{H 1})-(\mathbf{H} \mathbf{4})$ imply, that the function $(t, x, p) \rightarrow H(t, x, p)$ is continuous in $G \times \mathbb{R}^{n}$.

Let $(t, p) \in G, r \in \mathbb{R}^{n}, q \in \mathbb{R}^{n}$. Define sets:

$$
\begin{aligned}
& F(t, p)=\left\{f \in \mathbb{R}^{n+1}: f=(0, \dot{y}),|f| \leq \sqrt{2} L(t, p)\right\}, \\
& F_{g}(t, q, p)=\{f \in F(t, p):\langle-\dot{y}, q\rangle \geq H(t, q, p)\}, \\
& F_{d}(t, r, p)=\{f \in F(t, p):\langle-\dot{y}, r\rangle \leq H(t, r, p)\} .
\end{aligned}
$$


We easily see, that $F_{d}(t, r, p) \neq \emptyset, F_{g}(t, q, p) \neq \emptyset$. We observe also, that the sets $F_{g}(t, q, p), F_{p}(t, r, p)$ are convex and compact, and the multivalued mappings $(t, p) \rightarrow F_{g}(t, q, p),(t, p) \rightarrow F_{d}(t, r, p)$ are continuous.

Let us consider the differential inclusions $(\dot{p}(t)=(0, \dot{y}(t))$

$$
\begin{aligned}
& \dot{p}(t) \in F_{g}(t, q, p(t)), \\
& \dot{p}(t) \in F_{d}(t, r, p(t)) .
\end{aligned}
$$

Take $\left(t_{0}, p_{0}, q\right) \in[0, T] \times \mathbb{R}^{n+1} \times \mathbb{R}^{n}$. By $Y_{g}\left(t_{0}, p_{0}, q\right)$ denote a set of absolutely continuous functions $t \rightarrow p(t):[0, T] \rightarrow \mathbb{R}^{n+1}$, satisfying differential inclusion (13), for almost each $t \in[0, T]$, and such that $p\left(t_{0}\right)=p_{0}$. Analogously by $Y_{d}\left(t_{0}, p_{0}, r\right)$ denote a set of solutions to differential inclusion (14) satisfying the condition $p\left(t_{0}\right)=p_{0}$. By a known theorem on differential inclusion the sets $Y_{g}\left(t_{0}, p_{0}, q\right)$ and $Y_{d}\left(t_{0}, p_{0}, r\right)$ are nonempty and compact in $C\left([0, T], \mathbb{R}^{n+1}\right)$ - the space of continuous functions with the norm $\|p(\cdot)\|=\max _{t \in[0, T]}|p(t)|$.

Now we are able to define an upper and lower solutions to the problem (9), (8), (10).

Definition 1. An upper solution to the problem (9), (8), (10) we shall call a pair of functions $W(t, p), X(t, p),(t, p) \in \bar{G}$, such that for $V(t, p)=$ $W(t, p) y_{0}+X(t, p) y, V(\cdot, \cdot)$, is lower semicontinuous, $X(T, \cdot)$ is continuous, $y^{0} W(T, p) \geq y^{0} l(-X(T, p)), p \in \mathbb{R}^{n+1}$ and the inequality

$$
\sup _{\tau \in\left(t_{0}, T\right], q \in X c} \inf _{p(\cdot) \in Y_{g}\left(t_{0}, p_{0}, q\right)}\left[V(\tau, p(\tau))-V\left(t_{0}, p_{0}\right)\right] \leq 0,
$$

for $\left(t_{0}, p_{0}\right) \in \bar{G}$, where $\bar{G}=[0, T] \times \mathbb{R}^{n+1}$, holds.

Definition 2. A lower solution to the problem (9), (8), (10) we shall call a pair of functions $W(t, p), X(t, p), \quad(t, p) \in \bar{G}$, such that for $V(t, p)=$ $W(t, p) y_{0}+X(t, p) y, V(\cdot, \cdot)$, is upper semicontinuous, $X(T, \cdot)$ is continuous, $y^{0} W(T, p) \leq y^{0} l(-X(T, p)), p \in \mathbb{R}^{n+1}$ and the inequality

$$
\inf _{\tau \in\left(t_{0}, T\right], r \in X c} \sup _{p(\cdot) \in Y_{d}\left(t_{0}, p_{0}, r\right)}\left[V(\tau, p(\tau))-V\left(t_{0}, p_{0}\right)\right] \geq 0,
$$

for $\left(t_{0}, y_{0}\right) \in \bar{G}$, holds.

Definition 3. A minimax solution to the problem (9), (8), (10) we shall call a pair of functions $W(t, p), X(t, p),(t, p) \in \bar{G}$, such that for $V(t, p)=$ $W(t, p) y_{0}+X(t, p) y, V(\cdot, \cdot)$, is continuous and which are simultaneously a lower and an upper solution to that problem.

Since a minimax solution to the problem (9), (8), (10) is a pair of functions $W(t, p), X(t, p),(t, p) \in \bar{G}$ we should understand what each of these 
functions meant for primal problem i.e. for equation (5). In the case of (5) we look for a function $S(t, x)$ being solution to (5) (classical or generalized) in a given fixed domain $\Omega$ where the equation (5) is considered. In the case of (9), (8), (10) we also have a domain $\bar{G}$, but, in a dual space, so this domain is to some extend dual to $\Omega$. However our dual solution of (9), (8), (10) should also give a solution to (5) and as we can see from the introduction (see also Section 2.1 and [7]) that just the fuction $X(t, p)$ is corresponding for relation between sets $\Omega$ and $\bar{G}$ i.e.: $\bar{G} \ni(t, p) \rightarrow(t, X(t, p)) \in \Omega$. Therefore, if a such fuction $X(t, p)$ exists then we should consider it as a fixed in all deliberations concerning (9) (compare proof of Lemma 4, Corollary 5, Remark 1 and so on. Hence, we propose the following definition of uniqness for problem (9), (8), (10).

Definition 3' . A minimax solution to the problem (9), (8), (10) is unique if for a given $X(t, p), \quad(t, p) \in \bar{G}$ there exists exactly one function $W(t, p)$, $(t, p) \in \bar{G}$ such that the pair $W(t, p), X(t, p), \quad(t, p) \in \bar{G}$, is a minimax solution to the problem (9), (8), (10).

The sets of upper and lower solutions we shall denote by Sol $_{g}, S_{\text {ol }}$, respectively. If $(W, X) \in S l_{g}$ then the lower semicontinuous functional $p(\cdot) \rightarrow V(\tau, p(\tau))$ attains its minimum on the compact set $Y_{g}\left(t_{0}, p_{0}, q\right)$. That is why the inf in formula (15) can be replaced by the min. Analogously in formula (16) one can insert the max instead of the sup. Notice also that in view of the homogeneous assumption (H4) the restrictions $q \in X c$ and $r \in X c$ one can get rid of putting there $q \in \mathbb{R}^{n}, r \in \mathbb{R}^{n}$.

From the definitions of the sets (12) and (11) we easily obtain the following relations:

$$
\begin{aligned}
& F_{g}(t, q, p) \cap F_{d}(t, r, p) \neq \emptyset, \\
& \max _{q \in X c} \min _{f \in F_{g}(t, q, p), f=(0, \dot{y})}\langle-\dot{y}, x\rangle=H(t, x, p) \text {, } \\
& \min _{r \in X c} \max _{f \in F_{d}(t, r, p), f=(0, \dot{y})}\langle-\dot{y}, x\rangle=H(t, x, p) .
\end{aligned}
$$

\subsection{Relation of minimax solution to the classical one.}

In this section we show that a classical global solution of (9), (8), (10) (if it exists) is a minimax solution in the sense of the Definition 3.

Let $V(t, p)$ be a global of $C^{1}$ solution to $(9),(8),(10)$. First we show that it is an upper solution to that problem. Let us put $X(t, p)=V_{y}(t, p)$ and rewrite (9) to the equation

$$
V_{t}(t, p)+H(t,-X(t, p), p)=0 .
$$


Let $(t, p) \in G, q \in X c$ and define the set

$$
\begin{aligned}
F^{0}(t, q, p)= & \left\{f^{0} \in F_{g}(t, q, p): f^{0}=\left(0, \dot{y}_{0}\right),\right. \\
& \left.\left\langle-\dot{y}_{0},-X(t, p)\right\rangle=\min _{f \in F_{g}(t, q, p), f=(0, \dot{y})}\left\langle-\dot{y},-V_{y}(t, p)\right\rangle\right\} .
\end{aligned}
$$

In view of $(17)$

$$
\begin{aligned}
\left\langle-\dot{y}_{0},-X(t, p)\right\rangle \leq & H(t,-X(t, p), p), \\
& \text { for } f^{0} \in F_{g}(t, q, p), f^{0}=\left(0, \dot{y}_{0}\right) .
\end{aligned}
$$

From the definition of the set $F^{0}$ it is obvious that $F^{0}$ is nonempty, convex, compact subset of $F_{g}$. Since both multivalued mapping $(t, p) \rightarrow F_{g}(t, q, p)$ and $(t, p) \rightarrow X(t, p)$, are continuous the multivalued map $(t, p) \rightarrow F^{0}(t, q, p)$ is upper semicontinuous. Let us take any point $\left(t_{0}, p_{0}\right) \in[0, T) \times \mathbb{R}^{n+1}$ and denote by $Y^{0}\left(t_{0}, p_{0}, q\right)$ a set of solutions to the differential inclusion

$$
\dot{p}(t) \in F^{0}(t, q, p(t)), p\left(t_{0}\right)=p_{0} .
$$

Of course, $Y^{0}\left(t_{0}, p_{0}, q\right) \neq \emptyset$ and $Y^{0}\left(t_{0}, p_{0}, q\right) \subset Y_{g}\left(t_{0}, p_{0}, q\right)$.

Let $p(\cdot) \in Y^{0}\left(t_{0}, p_{0}, q\right)$ and define $t \rightarrow V(t, p(t)):[0, T] \rightarrow \mathbb{R}^{1}$. This function is absolutely continuous and its derivative (almost everywhere in $[0, T])$ satisfies, in view of (19) and (20) the estimation (we have in mind that $y^{0}=$ constant)

$$
d V(t, p(t)) / d t=\partial V(t, p(t)) / \partial t+\langle X(t, p(t)), \dot{y}(t)\rangle \leq 0 .
$$

The latter means that for all $t_{0}, p_{0}, \tau, q, \tau \in\left[t_{0}, T\right], p_{0} \in \mathbb{R}^{n+1}, q \in X c$, we have $V(\tau, p(\tau)) \leq V\left(t_{0}, p\left(t_{0}\right)\right)$, where $p(\cdot) \in Y^{0}\left(t_{0}, p_{0}, q\right) \subset Y_{g}\left(t_{0}, p_{0}, q\right)$. The function $V$ satisfies also conditions (8), (10) with $W(t, p)=V_{y_{0}}(t, p)$, thus the pair $(W, X)$ is an upper solution to $(9),(8),(10)$. Analogously one may show that the pair $(W, X)$ is a lower solution to $(9),(8),(10)$ and hence it is minimax solution.

Now we prove that at each point where the function $V$ of the minimax solution $(W, X)\left(V(t . p)=W(t, p) y_{0}+X(t, p) y\right)$ is differentiable it satisfies (9).

First consider an upper solution to (9), (8), (10). Let $(W, X) \in S_{\text {Sol }}$, $\left(t_{0}, p_{0}\right) \in G$. Assume that $V$ is differentiable at $\left(t_{0}, p_{0}\right)$. Take $\delta \in\left(0, T-t_{0}\right]$ and $q \in X c$. From (15) we infer that there is a solution $p_{\delta}(\cdot) \in Y_{g}\left(t_{0}, p_{0}, q\right)$ such that

$$
V\left(t_{0}+\delta, p_{\delta}\left(t_{0}+\delta\right)\right)-V\left(t_{0}, p_{0}\right) \leq 0 .
$$

From the continuity of $(t, p) \rightarrow F_{g}(t, q, p)$ we get

$$
p_{\delta}\left(t_{0}+\delta\right)=p_{0}+\left(f_{\delta}+g_{\delta}\right) \delta,
$$

where $f_{\delta} \in F_{g}\left(t_{0}, q, p_{0}\right),\left|g_{\delta}\right| \rightarrow 0$ if $\delta \rightarrow 0$. 
Since the set $F_{g}\left(t_{0}, q, p_{0}\right)$ is compact, there is a sequence $\delta_{k} \in\left(0, T-t_{0}\right]$, $k=1,2, \ldots$, such that

$$
\delta_{k} \rightarrow 0 \text { and } f_{\delta_{k}} \rightarrow f_{*} \in F_{g}\left(t_{0}, q, p_{0}\right) \text {, when } k \rightarrow \infty .
$$

From the differentiability of $V$ at $\left(t_{0}, p_{0}\right)$, taking into account (21), we have

$$
\begin{aligned}
& \frac{V\left(t_{0}+\delta_{k}, p_{\delta_{k}}\left(t_{0}+\delta_{k}\right)\right)-V\left(t_{0}, p_{0}\right)}{\delta_{k}} \\
& =\partial V\left(t_{0}, p_{0}\right) / \partial t+\left\langle X\left(t_{0}, p_{0}\right), f_{\delta_{k}}+g_{\delta_{k}}\right\rangle+a_{k} \leq 0,
\end{aligned}
$$

where $a_{k} \rightarrow 0$ when $k \rightarrow \infty$, and we assume that $X\left(t_{0}, p_{0}\right)=V_{y}\left(t_{0}, p_{0}\right)$. If we take a limit (when $k \rightarrow \infty$ ) in the left hand side of the last equality and take into account $(22)$ we obtain

$$
\begin{aligned}
& \partial V\left(t_{0}, p_{0}\right) / \partial t+\underset{f \in F_{g}\left(t_{0}, q, p_{0}\right), f=(0, \dot{y})}{\min }\left\langle X\left(t_{0}, p_{0}\right), \dot{y}\right\rangle \\
& \leq \partial V\left(t_{0}, p_{0}\right) / \partial t+\left\langle-X\left(t_{0}, p_{0}\right),-f_{*}\right\rangle \leq 0 .
\end{aligned}
$$

The last inequality holds for all $q \in X c$, hence we conclude that

$$
\begin{aligned}
& \partial V\left(t_{0}, p_{0}\right) / \partial t+H\left(t_{0},-V_{y}\left(t_{0}, p_{0}\right), p_{0}\right) \\
& \quad=\partial V\left(t_{0}, p_{0}\right) / \partial t+\max _{q \in X c} \min _{f \in F_{g}\left(t_{0}, q, p_{0}\right), f=(0, \dot{y})}\left\langle-X\left(t_{0}, p_{0}\right),-\dot{y}\right\rangle \leq 0 .
\end{aligned}
$$

Thus we come to the conclusion that at each point $\left(t_{0}, p_{0}\right) \in G$ at which an upper solution is differentiable it must satisfy inequality (23). Analogously one can show the converse inequality for a lower solution $(W, X)$. Therefore we have gotten that once the function $V$ of a minimax solution is differentiable at some point it satisfies the equation (9) at this point.

\subsection{Invariability of the definition of minimax solutions.}

In the definitions of the sets $F_{g}, F_{d}$ (see (12) and (11)) a certain indefiniteness is admitted, e.g. the definition of the function $L(t, p)$ is not unique. We show below that some indefiniteness does not influence the Definitions 1,2 , 3. Moreover, instead of maps $(t, p) \rightarrow F_{g}(t, q, p),(t, p) \rightarrow F_{d}(t, r, p)$ we can consider any maps $F_{g}, F_{d}$ satisfying conditions written below.

Let $P$ and $Q$ be nonempty sets in $\mathbb{R}^{n}$. Consider multivalued maps:

$$
\begin{aligned}
(t, q, p) & \rightarrow F_{g}(t, q, p) \subset \mathbb{R}^{n+1},(t, p, q) \in G \times Q, \\
(t, r, p) & \rightarrow F_{d}(t, r, p) \subset \mathbb{R}^{n+1},(t, p, r) \in G \times P .
\end{aligned}
$$

Assume the following hypotheses:

H'1 For each $(t, p, q, r) \in G \times Q \times P$ the sets $F_{g}(t, q, p)$ and $F_{d}(t, r, p)$ are nonempty, convex and compact; for any $t \in(0, T), r \in P, q \in Q$ and $f \in F_{g}(t, q, p) \cup F_{d}(t, r, p)$ the following estimation

$$
|f| \leq(1+|p|) c, c>0 \text { - any constant, }
$$


holds.

H'2 For each $(q, r) \in Q \times P$ multivalued maps $(t, p) \rightarrow F_{g}(t, q, p),(t, p) \rightarrow$ $F_{d}(t, r, p)$ are upper semicontinuous.

H'3 For each $(t, p) \in G$ and $x \in \mathbb{R}^{n}$

$$
\begin{aligned}
& \sup _{q \in Q} \min _{f \in F_{g}(t, q, p), f=(0, \dot{y})}\langle-\dot{y}, x\rangle=H(t, x, p), \\
& \inf _{r \in P} \max _{f \in F_{d}(t, r, p), f=(0, \dot{y})}\langle-\dot{y}, x\rangle=H(t, x, p) .
\end{aligned}
$$

From the Banach-Hahn theorem we get immediately

$$
F_{g}(t, q, p) \cap F_{d}(t, r, p) \neq \emptyset, \text { for }(t, p, q, r) \in G \times Q \times P .
$$

The set of all $F_{g}$ (respectively $F_{d}$ ) satisfying $\left(\mathbf{H}^{\prime} \mathbf{1}\right)-\left(\mathbf{H}^{\prime} \mathbf{3}\right)$ will be denoted by $\mathbf{F}_{g}(H)$ (respectively $\mathbf{F}_{d}(H)$ ). If a Hamiltonian satisfies $(\mathbf{H 1})-(\mathbf{H} \mathbf{3})$ then $\mathbf{F}_{g}(H) \neq \emptyset$ and $\mathbf{F}_{d}(H) \neq \emptyset$. In particular, multivalued maps $F_{g}, F_{d}$ defined in (12) and (11) satisfy hypotheses (H'1)-(H'3).

Let us take any $F_{g} \in \mathbf{F}_{g}(H)$. We can thus use in differential inclusion (13) that $F_{g}$. What means, that for any $F_{g} \in \mathbf{F}_{g}(H)$ we are able to define a set $\operatorname{Sol}_{g}\left(F_{g}\right)$ consisting of all pairs of functions $W(t, p), X(t, p),(t, p) \in \bar{G}$, such that $V(\cdot, \cdot), V(t, p)=W(t, p) y^{0}+X(t, p) y$, is lower semicontinuous, $X(T, \cdot)$ is continuous, $W(T, p) y^{0} \geq y^{0} l(-X(T, p)), p=\left(y^{0}, y\right) \in \mathbb{R}^{n+1}$, and that $V$ satisfies

$$
\sup _{\tau \in\left(t_{0}, T\right], q \in Q} \inf _{p(\cdot) \in Y_{g}\left(t_{0}, p_{0}, q\right)} V(\tau, p(\tau)) \leq V\left(t_{0}, p_{0}\right),
$$

for $\left(t_{0}, p_{0}\right) \in[0, T] \times \mathbb{R}^{n+1}$.

Analogously for any $F_{d} \in \mathbf{F}_{d}(H)$ we are able to define a set $\operatorname{Sol}_{d}\left(F_{d}\right)$ consisting of all pair of functions $W(t, p), X(t, p),(t, p) \in \bar{G}$, such that $V(\cdot, \cdot), V(t, p)=W(t, p) y^{0}+X(t, p) y$, is upper semicontinuous, $X(T, \cdot)$ is continuous, $W(T, p) y^{0} \leq y^{0} l(-X(T, p)), p=\left(y^{0}, y\right) \in \mathbb{R}^{n+1}$ and that $V$ satisfies

$$
\inf _{\tau \in\left(t_{0}, T\right], r \in P} \sup _{p(\cdot) \in Y_{d}\left(t_{0}, p_{0}, r\right)} V(\tau, p(\tau)) \geq V\left(t_{0}, p_{0}\right),
$$

for $\left(t_{0}, p_{0}\right) \in[0, T] \times \mathbb{R}^{n+1}$.

In the next section we proof, that for any pair $\left(F_{g}, F_{d}\right) \in \mathbf{F}_{g}(H) \times \mathbf{F}_{d}(H)$ the intersection $\operatorname{Sol}_{g}\left(F_{g}\right) \cap \operatorname{Sol}_{d}\left(F_{d}\right)$ contains exactly one pair of functions $(W, X)$. Moreover this pair does not depend on choice of $F_{g} \in \mathbf{F}_{g}(H)$ and $F_{d} \in \mathbf{F}_{d}(H)$. The latter means, in order to define a minimax solution of (9), (8), (10), we may use any $F_{g} \in \mathbf{F}_{g}(H), F_{d} \in \mathbf{F}_{d}(H)$. 


\section{Existence and uniqueness of minimax solution}

The proof of existence and uniqueness of minimax solutions will be divided into two parts. First, we shall consider minimum upper and maximum lower solutions. We prove that they exist and that minimum upper solution is less than or equal to maximum lower solution. Next we prove that any lower solution is less than or equal to any upper solution. These will allow us to infer the existence and uniqueness of solutions to our problem.

\subsection{Lower envelope of upper solutions.}

Let us take $F_{g} \in \mathbf{F}_{g}(H)$ and $F_{d} \in \mathbf{F}_{d}(H)$. Denote by $\Phi_{g}$ the set of pairs of functions $(W, X) ; W: \bar{G} \rightarrow[-\infty, \infty), X: \bar{G} \rightarrow \mathbb{R}^{n}, X(T, \cdot)-$ continuous, $W$ satisfies the inequality $W(T, p) y^{0} \geq y^{0} l(-X(T, p)), p \in \mathbb{R}^{n+1}$ and $V(t, p)=W(t, p) y^{0}+X(t, p) y$ the condition (15), by $\Phi_{d}$ it will be denoted the set of pairs of functions $(W, X) ; W: \bar{G} \rightarrow(-\infty, \infty], X: \bar{G} \rightarrow \mathbb{R}^{n}, X(T, \cdot)-$ continuous, $W$ satisfies the inequality $W(T, p) y^{0} \leq y^{0} l(-X(T, p)), p \in \mathbb{R}^{n+1}$ and $V(t, p)=W(t, p) y^{0}+X(t, p) y$ the condition (16).

The functions considered admit the values $-\infty$ and $+\infty$. Thus we assume: if $V\left(t_{0}, p_{0}\right)=+\infty$ then in the condition $(27) V(\tau, p(\tau)) \leq V\left(t_{0}, p_{0}\right)$ for all values of $V(\tau, p(\tau)) \in(-\infty, \infty]$, if $V\left(t_{0}, p_{0}\right)=-\infty$ then in the condition $(28) V(\tau, p(\tau)) \geq V\left(t_{0}, p_{0}\right)$ for all values of $V(\tau, p(\tau)) \in[-\infty, \infty)$. Note also that, we do not require that the function $V(t, p)=W(t, p) y^{0}+$ $X(t, p) y(W, X) \in \Phi_{g}\left((W, X) \in \Phi_{d}\right)$ is lower (upper) semicontinuous. Hence $\operatorname{Sol}_{g}\left(F_{g}\right) \subset \Phi_{g}$ and $\operatorname{Sol}_{d}\left(F_{d}\right) \subset \Phi_{d}$.

Set

$$
\begin{aligned}
& D_{g}\left(t_{0}, p_{0}, \tau, q\right)=\left\{p(\tau): p(\cdot) \in Y_{g}\left(t_{0}, p_{0}, q\right)\right\}, \\
& D_{d}\left(t_{0}, p_{0}, \tau, r\right)=\left\{p(\tau): p(\cdot) \in Y_{d}\left(t_{0}, p_{0}, r\right)\right\},
\end{aligned}
$$

where $\left(t_{0}, p_{0}\right) \in[0, T) \times \mathbb{R}^{n+1}, \tau \in\left[t_{0}, T\right], r \in P, q \in Q, Y_{g}\left(t_{0}, p_{0}, q\right)$ and $Y_{d}\left(t_{0}, p_{0}, r\right)$ are defined above.

The set of solutions of $p(\cdot):\left[t_{*}, T\right] \rightarrow \mathbb{R}^{n+1}$ of the differential inclusion

$$
\dot{p}(t) \in F(t, p(t))
$$

will be denoted by $Y\left(t_{*}, p_{*}\right)$, here $F(t, p)=\left\{f \in \mathbb{R}^{n+1}: f=(0, \dot{y}),|f| \leq\right.$ $(1+|p|) c\},($ see $(24)),\left(t_{*}, p_{*}\right) \in[0, T) \times \mathbb{R}^{n+1}$ - a given point, and $p(\cdot) \in$ $Y\left(t_{*}, p_{*}\right)$ satisfies the initial condition $p\left(t_{*}\right)=p_{*}$. Denote by $D\left(t_{*}, p_{*}\right)$ a set covered by the graphs of trajectories of $p(\cdot) \in Y\left(t_{*}, p_{*}\right)$. The five lemmas below will be enough to infer that $\Phi_{g} \cap \Phi_{d} \neq \emptyset$. 
Lemma 1. For any pair $(W, X) \in \Phi_{g}, q_{*} \in Q$ and $\left(t_{0}, p_{0}\right) \in \bar{G}$, for $V(t, p)=W(t, p) y^{0}+X(t, p) y$ the following estimation

$$
\begin{aligned}
& V\left(t_{0}, p_{0}\right) \geq v_{-}\left(t_{0}, p_{0}, q_{*}, X\right) \\
& =\min \left\{X(T, p) y+y^{0} l(-X(T, p)): p \in D_{g}\left(t_{0}, p_{0}, T, q_{*}\right)\right\}
\end{aligned}
$$

holds.

Proof. Let us put $\tau=T$ in (27), then we have

$$
\begin{aligned}
& V\left(t_{0}, p_{0}\right) \geq \inf \left\{V(T, p(T)): p(\cdot) \in Y_{g}\left(t_{0}, p_{0}, q_{*}\right)\right\} \\
& =\inf \left\{V(T, p): p \in D_{g}\left(t_{0}, p_{0}, T, q_{*}\right)\right\} \\
& \geq \min \left\{y^{0} l(-X(T, p))+X(T, p) y: p \in D_{g}\left(t_{0}, p_{0}, T, q_{*}\right)\right\} \\
& =v_{-}\left(t_{0}, p_{0}, q_{*}, X\right) .
\end{aligned}
$$

The last inequality follows from the fact that $W(T, p) \geq y^{0} l(-X(T, p))$. We know that the set $D_{g}\left(t_{0}, p_{0}, T, q_{*}\right)$ is compact in $\mathbb{R}^{n+1}$, and if $t_{0}=T$, then $D_{g}\left(t_{0}, p_{0}, T, q_{*}\right)=\left\{p_{0}\right\}$, thus

$$
v_{-}\left(T, p, q_{*}, X\right)=X(T, p) y+y^{0} l(-X(T, p)), p \in \mathbb{R}^{n+1} .
$$

The function $p \rightarrow X(T, p) y+y^{0} l(-X(T, p))$ is lower semicontinuous, therefore it attains its minimum on $D_{g}\left(t_{0}, p_{0}, T, q_{*}\right)$. Hence the proof of lemma is ended.

Let $\theta \in[0, T], r \in P,\left(W_{*}, X_{*}\right) \in \Phi_{g}, V_{*}(t, p)=W_{*}(t, p) y^{0}+X_{*}(t, p) y$. For $p_{0} \in \mathbb{R}^{n+1}$, define

$$
\begin{aligned}
& V^{*}\left(t_{0}, p_{0}\right)= \begin{cases}V_{*}\left(t_{0}, p_{0}\right) & \text { if } \theta<t_{0} \leq T, \\
\sup \left\{V_{*}(\theta, p): p \in D_{d}\left(t_{0}, p_{0}, \theta, r\right)\right\} & \text { if } 0 \leq t_{0} \leq \theta,\end{cases} \\
& W^{*}\left(t_{0}, p_{0}\right) y_{0}^{0}=V^{*}\left(t_{0}, p_{0}\right)-X_{*}\left(t_{0}, p_{0}\right) y_{0} .
\end{aligned}
$$

Lemma 2. The pair $\left(W^{*}, X_{*}\right) \in \Phi_{g}$.

Proof. Since $\left(W_{*}, X_{*}\right)$ satisfies $W_{*}(T, p) \geq y^{0} l\left(-X_{*}(T, p)\right), p \in \mathbb{R}^{n+1}$, the pair $\left(W^{*}, X_{*}\right)$ satisfies it too. From $(34)$ we see that $V^{*}\left(t_{0}, p_{0}\right) \in(-\infty, \infty]$, $\left(t_{0}, p_{0}\right) \in \bar{G}$. We show that for $V^{*}$ the condition (27) is satisfied, i.e. for any $\varepsilon>0, t_{0} \in[0, T), p_{0} \in \mathbb{R}^{n+1}, \tau \in\left[t_{0}, T\right], q \in Q$ there is $p(\cdot) \in Y_{g}\left(t_{0}, p_{0}, q\right)$ such that

$$
V^{*}(\tau, p(\tau)) \leq V^{*}\left(t_{0}, p_{0}\right)+\varepsilon .
$$

Let us consider three cases: 
1. Let $t_{0} \in[\theta, T) . V_{*}$ satisfies $(27)$, therefore for $\theta<t_{0}<T$ the function $V^{*}$ satisfies that condition too. If $t_{0}=\theta$, then $D_{d}\left(t_{0}, p_{0}, \theta, r\right)=\left\{p_{0}\right\}$ and $V^{*}\left(t_{0}, p_{0}\right)=V_{*}\left(t_{0}, p_{0}\right)$. Hence $V^{*}$ satisfies (27) for $\theta \leq t_{0}<T$.

2. Let now $t_{0} \in[0, \theta)$ and $\tau \in\left[t_{0}, \theta\right]$. As the multivalued map $(t, p) \rightarrow$ $F_{g}(t, q, p) \cap F_{d}(t, r, p)$ has nonempty, convex and compact values, therefore $Y_{g}\left(t_{0}, p_{0}, q\right) \cap Y_{d}\left(t_{0}, p_{0}, r\right) \neq \emptyset$.

Take $p(\cdot) \in Y_{g}\left(t_{0}, p_{0}, q\right) \cap Y_{d}\left(t_{0}, p_{0}, r\right)$. From the definition of the set (30) we get

$$
p(\tau) \in D_{d}\left(t_{0}, p_{0}, \tau, r\right), \quad D_{d}(\tau, p(\tau), \theta, r) \subset D_{d}\left(t_{0}, p_{0}, \theta, r\right) .
$$

This is why, in view of (34), we have

$$
\begin{aligned}
V^{*}(\tau, p(\tau)) & =\sup \left\{V_{*}(\theta, p): p \in D_{d}(\tau, p(\tau), \theta, r)\right\} \\
& \leq V^{*}\left(t_{0}, p_{0}\right)=\sup \left\{V_{*}(\theta, p): p \in D_{d}\left(t_{0}, p_{0}, \theta, r\right)\right\} .
\end{aligned}
$$

Thus the condition (36) holds (for $\varepsilon=0$ ).

3. Let now $t_{0} \in[0, \theta)$ and $\tau \in(\theta, T]$. As we proved in the cases 1. and 2., there is a $p_{*}(\cdot) \in Y_{g}\left(t_{0}, p_{0}, q\right)$ and $p^{*}(\cdot) \in Y_{g}\left(\theta, p_{*}(\theta), q\right)$, such that

$$
V^{*}\left(\theta, p_{*}(\theta)\right) \leq V^{*}\left(t_{0}, p_{0}\right), V^{*}\left(\tau, p^{*}(\tau)\right) \leq V^{*}\left(\theta, p_{*}(\theta)\right)+\varepsilon .
$$

Put

$$
p(t)= \begin{cases}p_{*}(t), & 0 \leq t \leq \theta, \\ p^{*}(t), & \theta<t \leq T .\end{cases}
$$

We obtain $p(\cdot) \in Y_{g}\left(t_{0}, p_{0}, q\right)$ thus the condition (36) holds and the proof of the lemma is finished.

Lemma 3. The set $\Phi_{g}$ is nonempty and for any function $X: \bar{G} \rightarrow \mathbb{R}^{n}$, $X(T, \cdot)$ - conntinuous, $\Phi_{g}$ contains the pair of functions $\left(W_{+}, X\right)$, where $W_{+}(t, p) y^{0}=v_{+}\left(t, p, r_{*}, X\right)-X(t, p) y$ and $(t, p) \rightarrow v_{+}\left(t, p, r_{*}, X\right): \bar{G} \rightarrow \mathbb{R}$, is defined by the formula

$v_{+}\left(t_{0}, p_{0}, r_{*}, X\right)=\max \left\{X(T, p) y+y^{0} l(-X(T, p)): p \in D_{d}\left(t_{0}, p_{0}, T, r_{*}\right)\right\}$, where $\left(t_{0}, p_{0}\right) \in \bar{G}, r_{*}$ any point from $P$.

Proof. If $t_{0}=T$, then $D_{d}\left(t_{0}, p_{0}, T, r_{*}\right)=\left\{p_{0}\right\}$. Therefore

$$
v_{+}\left(T, p, r_{*}, X\right)=X(T, p) y+y^{0} l(-X(T, p)), p \in \mathbb{R}^{n+1} .
$$

Repeating the arguments from the proof of Lemma 2 (the case 2 .) we obtain that the function $v_{+}$satisfies (27). Hence we have proved the lemma. 
Lemma 4. Let

$$
\begin{gathered}
V_{0}(t, p, X)=\inf \left\{V(t, p): V(t, p)=W(t, p) y^{0}+X(t, p) y,(W, X) \in \Phi_{g}\right\}, \\
(t, p) \in \bar{G} .
\end{gathered}
$$

Then for each $(W, X) \in \Phi_{g}$ the pair $\left(W_{0}(X), X\right) \in \Phi_{g}$ with $W_{0}(t, p, X) y^{0}=$ $V_{0}(t, p, X)-X(t, p) y$ and $\left(W_{0}(X), X\right) \in \Phi_{d}$, and the following relations:

$$
\begin{gathered}
V_{0}(T, p, X)=X(T, p) y+y^{0} l(-X(T, p)), \\
v_{-}\left(t, p, q_{*}, X\right) \leq V_{0}(t, p, X) \leq v_{+}\left(t, p, r_{*}, X\right),
\end{gathered}
$$

for $t \in[0, T], p \in \mathbb{R}^{n+1}, q_{*} \in Q, \quad r_{*} \in P$, holds.

Proof. Relations (39), (40) we obtain at once by Lemmas 1, 3 and their proofs. We must still prove that the pairs $\left(W_{0}(X), X\right)$ satisfies $(27)$, and the condition (28).

To this effect let $\left(t_{0}, p_{0}, q\right) \in[0, T] \times \mathbb{R}^{n+1} \times Q, \tau \in\left[t_{0}, T\right], \varepsilon>0$. By the definition of inferior there exists a $(V, X) \in \Phi_{g}$ such that $V\left(t_{0}, p_{0}\right) \leq$ $V_{0}\left(t_{0}, p_{0}, X\right)+\varepsilon / 2$. The condition (27), which the function $V$ satisfies, implies that $V(\tau, p(\tau)) \leq V\left(t_{0}, p_{0}\right)+\varepsilon / 2$, where $p(\cdot)$ is an element of the set $Y_{g}\left(t_{0}, p_{0}, q\right)$. From (38) we also have the estimation $V_{0}(\tau, p(\tau), X) \leq$ $V(\tau, p(\tau))$. Therefore there exists a solution $p(\cdot) \in Y_{g}\left(t_{0}, p_{0}, q\right)$ such that $V_{0}(\tau, p(\tau), X) \leq V_{0}\left(t_{0}, p_{0}, X\right)+\varepsilon$, i.e. the function $V_{0}(X)$ satisfies the condition $(27)$.

Take now $\left(t_{0}, p_{0}, r\right) \in[0, T] \times \mathbb{R}^{n+1} \times P, \theta \in\left[t_{0}, T\right]$, define a function $V^{*}$ and $W^{*}$ by the formulae (34) and (35), in which we assume $V_{*}=V_{0}(X)$. According to the lemma 2 and the first part of its proof we have $\left(W^{*}, X\right) \in$ $\Phi_{g}$. Hence and from (38) we obtain $V^{*}\left(t_{0}, p_{0}\right) \geq V_{0}\left(t_{0}, p_{0}, X\right)$. Taking into account (34), in which we assume $V_{*}=V_{0}(X)$, the last estimation may be rewritten in the form:

$$
\begin{aligned}
V^{*}\left(t_{0}, p_{0}\right) & =\sup \left\{V_{0}(\theta, p, X): p \in D_{d}\left(t_{0}, p_{0}, \theta, r\right)\right\} \\
& =\sup \left\{V_{0}(\theta, p(\theta), X): p(\cdot) \in Y_{d}\left(t_{0}, p_{0}, r\right)\right\} \geq V_{0}\left(t_{0}, p_{0}, X\right) .
\end{aligned}
$$

The inequality obtained denotes that the function $V_{0}$ satisfies (28). Therefore Lemma 4 is proved.

A direct consequence of the above lemma is the following corollary.

Corollary 5. Let $V_{0}(X)$ and $W_{0}(X)$ be defined as in Lemma 4. Then the pair $\left(W_{0}(X), X\right) \in \Phi_{g} \cap \Phi_{d}$.

Remark 1. Let us notice that the function $V_{0}$ and, in consequence and $W_{0}$, are determined by $X$ in a unique way. If we take different $X$ we may obtain different $V_{0}$ and $W_{0}$. 
Let us recall that in the definition of the sets $\Phi_{d}$ and $\Phi_{g}$ we do not assume semicontinuity of the function $V$. Now we introduce two operators of closure: from above and below, and in consequence we construct an upper and a lower solution.

Let $Z: \bar{G} \rightarrow \mathbb{R}$ be any function. Put

$$
Z_{-}(t, p)=\liminf _{(s, z) \rightarrow(t, p)} Z(s, z), Z_{+}(t, p)=\limsup _{(s, z) \rightarrow(t, p)} Z(s, z) .
$$

Of course the functions $Z_{-}, Z_{+}$are lower and upper semicontinuous, respectively.

Lemma 6. Let $(W, X) \in \Phi_{g}$ and $V(t, p)=W(t, p) y^{0}+X(t, p) y<\infty$ for $(t, p) \in \bar{G}$, then for $W_{-}(t, p)=V_{-}(t, p) y^{0}-X(t, p) y\left(V_{-}\right.$is closure of $\left.V\right)$, $\left(W_{-}, X\right) \in$ Sol $_{g}$. Let $(W, X) \in \Phi_{d}$ and $V(t, p)=W(t, p) y^{0}+X(t, p) y>-\infty$ for $(t, p) \in \bar{G}$, then for $W_{+}(t, p)=V_{+}(t, p) y^{0}-X(t, p) y$ ( $V_{+}$is closure of $V),\left(W_{+}, X\right) \in S_{\text {Sol }}$.

Proof. Take any $(W, X) \in \Phi_{g}$ with $V(t, p)=W(t, p) y^{0}+X(t, p) y<\infty$ for $(t, p) \in \bar{G}$. From $(41)$ we have that $V_{-}(t, p) \leq V(t, p)<\infty$. From Lemma 1 and (41) we have $V_{-}(t, p) \geq v_{-}\left(t, p, q_{*}, X\right)>-\infty$, thus $V_{-}(t, p) \in(-\infty, \infty)$, for $(t, p) \in \bar{G}$.

We show that $V_{-}\left(T, p_{*}\right) \geq X\left(T, p_{*}\right) y_{*}+y_{*}^{0} l\left(-X\left(T, p_{*}\right)\right), p_{*} \in \mathbb{R}^{n+1}$. By the definition of the lower limit there exists a sequence $\left(t_{k}, p_{k}\right) \in \bar{G},(k=$ $1,2, \ldots)$ such that $\left(t_{k}, p_{k}\right) \rightarrow\left(T, p_{*}\right)$, if $k \rightarrow \infty$. As $(W, X) \in \Phi_{g}$, so $V$ satisfies (27). Let us chose $q \in Q$ and corresponding to it the solution $p^{k}(\cdot) \in Y_{g}\left(t_{k}, p_{k}, q\right)$, such that

$$
V\left(T, p^{k}(T)\right) \leq V\left(t_{k}, p_{k}\right)+1 / k .
$$

Let us recall that $V\left(T, p^{k}(T)\right) \geq X\left(T, p^{k}(T)\right) y^{k}(T)+y^{0 k}(T) l\left(-X\left(T, p^{k}(T)\right)\right)$. Let us calculate a limit when $k \rightarrow \infty$ in the last inequality. Taking into account that $p^{k}(T) \rightarrow p_{*}$, that the functions $X(T, \cdot)$ and $l$ are continuous, we obtain $V_{-}\left(T, p_{*}\right) \geq X\left(T, p_{*}\right) y_{*}+y_{*}^{0} l\left(-X\left(T, p_{*}\right)\right)$.

Next we show that $V_{-}$satisfies $(27)$. Let $\left(t_{0}, p_{0}, q\right) \in[0, T) \times \mathbb{R}^{n+1} \times Q$, $\tau \in\left[t_{0}, T\right]$. Choose a sequence $\left(t_{k}, p_{k}\right) \in \bar{G},(k=1,2, \ldots)$ such that

$$
\left(t_{k}, p_{k}\right) \rightarrow\left(t_{0}, p_{0}\right), \quad V\left(t_{k}, p_{k}\right) \rightarrow V_{-}\left(t_{0}, p_{0}\right), k \rightarrow \infty .
$$

The function $V$ satisfies $(27)$, hence there exists a solution $p^{k}(\cdot) \in Y_{g}\left(t_{k}, p_{k}, q\right)$, such that

$$
V\left(\tau, p^{k}(\tau)\right) \leq V\left(t_{k}, p_{k}\right)+1 / k .
$$

From the sequence $\left\{p^{k}(\cdot)\right\}$ we can chose a subsequence convergent uniformly to some function, which we denote by $p^{*}(\cdot)$. This function belongs 
to $Y_{g}\left(t_{0}, p_{0}, q\right)$. For a convenience we assume that $\left\{p^{k}(\cdot)\right\}$ is just that subsequence, i.e. $p^{k}(\cdot) \rightarrow p^{*}(\cdot)$, if $k \rightarrow \infty$. By the definition of the lower limit

$$
\liminf _{k \rightarrow \infty} V\left(\tau, p^{k}(\tau)\right) \geq V_{-}\left(\tau, p^{*}(\tau)\right) .
$$

Taking limits in (42), (43) with $k \rightarrow \infty$, we get

$$
V_{-}\left(\tau, p^{*}(\tau)\right) \leq \liminf _{k \rightarrow \infty} V\left(\tau, p^{k}(\tau)\right) \leq \lim _{k \rightarrow \infty}\left[V\left(t_{k}, p_{k}\right)+1 / k\right]=V_{-}\left(t_{0}, p_{0}\right),
$$

where $p^{*}(\cdot) \in Y_{g}\left(t_{0}, p_{0}, q\right)$. Thus $V_{-}$satisfies $(27)$.

From the above argumentation we see that the pair $\left(W_{-}, X\right)$ is an upper solution of our dual problem.

Analogously one can prove the second part of the lemma.

From Lemmas 4 and 6 we get that $\left(W_{0-}(X), X\right) \in \operatorname{Sol}_{g}$ and $\left(W_{0+}(X), X\right) \in$ $S o l_{d}$. By the definition of lower and upper semicontinuity we have $V_{0-}(t, p, X)$ $\leq V_{0+}(t, p, X)$ for $(t, p) \in \bar{G}$. Hence one obtains the following lemma.

Lemma 7. There exists a pair $\left(\left(W_{g}, X\right),\left(W_{d}, X\right)\right) \in$ Sol $_{g} \times$ Sol $_{d}$, such that the corresponding functions $V_{g}(t, p)=W_{g}(t, p) y^{0}+X(t, p) y$ and $V_{d}(t, p)=$ $W_{d}(t, p) y^{0}+X(t, p) y$ satisfy the inequality

$$
V_{g}(t, p) \leq V_{d}(t, p) \quad \text { for }(t, p) \in \bar{G} .
$$

\subsection{The existence and uniqueness of the solution.}

Let $\left(\left(W_{g}, X_{g}\right),\left(W_{d}, X_{d}\right)\right) \in \operatorname{Sol}_{g} \times S_{\text {Sol }}$. Since $(t, p) \rightarrow V_{g}(t, p)=$ $W_{g}(t, p) y^{0}+X_{g}(t, p) y$ is lower semicontinuous and $(t, p) \rightarrow V_{d}(t, p)=$ $W_{d}(t, p) y^{0}+X_{d}(t, p) y$ is upper semicontinuous, and the sets $Y_{g}\left(t_{0}, p_{0}, q\right)$, $Y_{d}\left(t_{0}, p_{0}, r\right)$ are compact in $C\left(\left[t_{0}, T\right], \mathbb{R}^{n+1}\right)$, therefore the conditions $(27)$, (28) we may replace with the following conditions:

$$
\begin{array}{ll}
\sup _{q \in Q} & \min _{p(\cdot) \in Y_{g}\left(t_{0}, p_{0}, q\right)} V_{g}(\tau, p(\tau)) \leq V_{g}\left(t_{0}, p_{0}\right), \\
\inf _{r \in P} & \max _{p(\cdot) \in Y_{d}\left(t_{0}, p_{0}, r\right)} V_{d}(\tau, p(\tau)) \geq V_{d}\left(t_{0}, p_{0}\right) .
\end{array}
$$

The existence and uniqueness of the solution will follow from Lemmas 7 and 8 proved below.

Lemma 8. For each $F_{g} \in \mathbf{F}_{g}(H), F_{d} \in \mathbf{F}_{d}(H)$ and each $\left(\left(W_{g}, X\right),\left(W_{d}, X\right)\right)$ $\in \operatorname{Sol}_{g}\left(F_{g}\right) \times \operatorname{Sol}_{d}\left(F_{d}\right)$ (with the same $X$ ) and $\left(t_{0}, p_{0}\right) \in \bar{G}$ the following inequality

$$
V_{g}\left(t_{0}, p_{0}\right) \geq V_{d}\left(t_{0}, p_{0}\right)
$$

holds. 
Proof. We shall investigate the sets

$$
\begin{aligned}
W_{a}= & \left\{\left(p_{g}(\cdot), p_{d}(\cdot)\right) \in Y\left(t_{0}, p_{0}\right) \times Y\left(t_{0}, p_{0}\right):\right. \\
& \left.\langle s(t), \dot{s}(t)\rangle \leq \Lambda|s(t)|^{2}+a \text { for a.e. } t \in\left[t_{0}, T\right]\right\},
\end{aligned}
$$

where $s(t)=y_{d}(t)-y_{g}(t)$, (we have in mind that the first coordinate of $p_{g}$ and $p_{d}$ is constant function of $t$ and equal to $\left.y_{0}^{0}, p_{0}=\left(y_{0}^{0}, y_{0}\right)\right) a-\mathrm{a}$ positive number, $\Lambda$ - Lipschitz constant from (H2) with

$$
\begin{aligned}
E= & \left\{\left(t, p_{*}\right) \in\left[t_{0}, T\right] \times \mathbb{R}^{n}:\right. \\
& \text { there exists } \left.p(\cdot) \in Y\left(t_{0}, p_{0}\right), \text { such that } p(t)=p_{*}\right\} .
\end{aligned}
$$

Let us put

$$
\begin{aligned}
M_{a}(t)= & \left\{\left(p_{g}(\cdot), p_{d}(\cdot)\right) \in W_{a}:\right. \\
& \left.V_{g}\left(t_{0}, p_{0}\right) \geq V_{g}\left(t, p_{g}(t)\right), V_{d}\left(t_{0}, p_{0}\right) \leq V_{d}\left(t, p_{d}(t)\right)\right\}, \\
t_{a}= & \sup \left\{t \in\left[t_{0}, T\right]: M_{a}(t) \neq \emptyset\right\} .
\end{aligned}
$$

Notice that $W_{a}$ is a compact set, and the functions $V_{g}, V_{d}$ are lower and upper semicontinuous, respectively. This is why superior in (50) is attained.

We prove that for any $a>0$ the equality

$$
t_{a}=T
$$

holds.

First we show that the inequality (46) follows from (51). To this effect let us consider a sequence $\left(p_{g}^{k}(\cdot), p_{d}^{k}(\cdot)\right) \in M_{a_{k}}(T), k=1,2, \ldots$, where $a_{k} \rightarrow 0$, when $k \rightarrow \infty$. By (49) we get

$$
\begin{aligned}
V_{g}\left(t_{0}, p_{0}\right) & \geq V_{g}\left(T, p_{g}^{k}(T)\right) \\
& \geq X\left(T, p_{g}^{k}(T)\right) y_{g}^{k}(T)+y_{g}^{0 k}(T) l\left(-X\left(T, p_{g}^{k}(T)\right),\right. \\
V_{d}\left(t_{0}, p_{0}\right) & \leq V_{d}\left(T, p_{d}^{k}(T)\right) \\
& \leq X\left(T, p_{d}^{k}(T)\right) y_{d}^{k}(T)+y_{d}^{0 k}(T) l\left(-X\left(T, y_{d}^{k}(T)\right) .\right.
\end{aligned}
$$

Without loss of generality we can assume that $p_{g}^{k}(\cdot) \rightarrow p_{g}^{*}(\cdot), p_{d}^{k}(\cdot) \rightarrow p_{d}^{*}(\cdot)$, when $k \rightarrow \infty$. From the definition of $W_{a}$ we get that $p_{g}^{*}(\cdot)=p_{d}^{*}(\cdot)$. Thus taking a limit in (52) with $k \rightarrow \infty$, we obtain (46).

Therefore to end the proof we must show that (51) holds. On the contrary, let us assume that $t_{a}<T$. Let $t^{0}=t_{a},\left(p_{g}^{0}(\cdot), p_{d}^{0}(\cdot)\right) \in M_{a}\left(t^{0}\right)$. Set $p_{g}^{0}=$ $\left(y_{0}^{0}, y_{g 0}\right)=p_{g}^{0}\left(t^{0}\right), p_{d}^{0}=\left(y_{0}^{0}, y_{d 0}\right)=p_{d}^{0}\left(t^{0}\right), s^{0}=y_{d 0}-y_{g 0}$. From (49) we obtain the inequality

$$
V_{g}\left(t_{0}, p_{0}\right) \geq V_{g}\left(t^{0}, p_{g}^{0}\right), \quad V_{d}\left(t_{0}, p_{0}\right) \leq V_{d}\left(t^{0}, p_{d}^{0}\right) .
$$


Using (H'3), let us choose $r_{a} \in P$ and $q_{a} \in Q$ such that

$$
\begin{aligned}
& \left\langle s^{0}, \dot{y}_{g}\right\rangle \geq H\left(t^{0},-s^{0}, p_{g}^{0}\right)-a / 4, f_{g} \in F_{g}\left(t^{0}, q_{a}, p_{g}^{0}\right), f_{g}=\left(0, \dot{y}_{g}\right), \\
& \left\langle s^{0}, \dot{y}_{d}\right\rangle \leq H\left(t^{0},-s^{0}, p_{d}^{0}\right)-a / 4, f_{d} \in F_{d}\left(t^{0}, r_{a}, p_{d}^{0}\right), f_{d}=\left(0, \dot{y}_{d}\right) .
\end{aligned}
$$

As the maps $(t, p) \rightarrow F_{g}\left(t, q_{a}, p\right),(t, p) \rightarrow F_{d}\left(t, r_{a}, p\right)$ are upper semicontinuous and Hamiltonian $H$ is continuous, thus there exists $\delta>0$ such that for almost all $t \in\left[t^{0}, t^{0}+\delta\right]$ and for all $p_{g}(\cdot)=\left(y_{0}^{0}, y_{g}(\cdot)\right) \in Y_{g}\left(t^{0}, p_{g}^{0}, q_{a}\right)$, $p_{d}(\cdot)=\left(y_{0}^{0}, y_{d}(\cdot)\right) \in Y_{d}\left(t^{0}, p_{d}^{0}, r_{a}\right)$ the inequalities

$$
\begin{aligned}
& \left\langle s(t), \dot{y}_{g}(t)\right\rangle \geq H\left(t,-s(t), p_{g}(t)\right)-a / 2, \\
& \left\langle s(t), \dot{y}_{d}(t)\right\rangle \leq H\left(t,-s(t), p_{d}(t)\right)+a / 2
\end{aligned}
$$

holds, where $s(t)=y_{d}(t)-y_{g}(t)$. Let us notice that $\left(t, p_{d}(t)\right) \in E,\left(t, p_{g}(t)\right) \in$ $E, t^{0} \leq t \leq t^{0}+\delta$. From (H2) and (H4) we obtain

$$
\begin{aligned}
\langle s(t), \dot{s}(t)\rangle & =\left\langle s(t), \dot{y}_{d}(t)\right\rangle-\left\langle s(t), \dot{y}_{g}(t)\right\rangle \\
& \leq H\left(t,-s(t), p_{d}(t)\right)-H\left(t,-s(t), p_{g}(t)\right)+a \\
& \leq \Lambda|s(t)|^{2}+a .
\end{aligned}
$$

(44) and (45) imply that there exist $p_{g}^{\prime}(\cdot) \in Y_{g}\left(t^{0}, p_{g}^{0}, q_{a}\right)$ and $p_{d}^{\prime}(\cdot) \in$ $Y_{d}\left(t^{0}, p_{d}^{0}, r_{a}\right)$ such that

$$
\begin{aligned}
& V_{g}\left(t_{0}, p_{0}\right) \geq V_{g}\left(t^{0}, p_{g}^{0}\right) \geq V_{g}\left(t^{0}+\delta, p_{g}^{\prime}\left(t^{0}+\delta\right)\right), \\
& V_{d}\left(t_{0}, p_{0}\right) \leq V_{d}\left(t^{0}, p_{d}^{0}\right) \leq V_{d}\left(t^{0}+\delta, p_{d}^{\prime}\left(t^{0}+\delta\right)\right) .
\end{aligned}
$$

Let us put

$$
\begin{aligned}
& p_{g}(t)= \begin{cases}p_{g 0}(t), & t_{0} \leq t<t^{0}, \\
p_{g}^{\prime}(t), & t^{0} \leq t<t^{0}+\delta \\
p_{g}^{\prime}\left(t^{0}+\delta\right), & t^{0}+\delta \leq t \leq T,\end{cases} \\
& p^{\prime \prime}{ }_{d}(t)= \begin{cases}p_{d 0}(t), & t_{0} \leq t<t^{0}, \\
p_{d}^{\prime}(t), & t^{0} \leq t<t^{0}+\delta \\
p_{d}^{\prime}\left(t^{0}+\delta\right), & t^{0}+\delta \leq t \leq T .\end{cases}
\end{aligned}
$$

From the construction above we infer that $\left(p{ }_{g}(\cdot), p^{\prime \prime}{ }_{d}(\cdot)\right) \in M_{a}\left(t^{0}+\delta\right)$ (here $\left.t^{0}=t_{a}\right)$. In this way we come to a contradiction with the assumption $t_{a}<T$ - see the definition of $t_{a}(50)$. The proof of the lemma is finished.

The Lemmas 7 and 8 show that $\operatorname{Sol}_{g}\left(F_{g}\right) \cap \operatorname{Sol}_{d}\left(F_{d}\right) \neq \emptyset$. Corollary 5 and Remark 1 assert that this set contains only one element if we fix a function $X(t, p)$. Thus, that intersection for a fixed function $X(t, p)$ contains exactly one pair $(W, X)$. Moreover that pair does not depend on a choice of $F_{g} \in \mathbf{F}_{g}(H)$ and $F_{d} \in \mathbf{F}_{d}(H)$, if we take the same $X$. 
Indeed, let $F_{g}^{i} \in \mathbf{F}_{g}(H)$ and $F_{d}^{i} \in \mathbf{F}_{d}(H),\left(W_{i}, X\right) \in \operatorname{Sol}_{g}\left(F_{g}^{i}\right) \cap \operatorname{Sol}_{d}\left(F_{d}^{i}\right)$ $(i=1,2)$. Since $\left(W_{1}, X\right) \in \operatorname{Sol}_{g}\left(F_{g}^{1}\right)$ and $\left(W_{2}, X\right) \in \operatorname{Sol}_{d}\left(F_{d}^{2}\right)$, therefore by Lemma 8 we have $V_{1} \geq V_{2}\left(V_{1}(t, p)=W_{1}(t, p) y^{0}+X(t, p) y, V_{2}(t, p)=\right.$ $\left.W_{2}(t, p) y^{0}+X(t, p) y\right)$. On the other hand $\left(W_{1}, X\right) \in \operatorname{Sol}_{d}\left(F_{d}^{1}\right)$ and $\left(W_{2}, X\right) \in$ $\operatorname{Sol}_{g}\left(F_{g}^{2}\right)$, that is $V_{1} \leq V_{2}$. Hence $V_{1}=V_{2}$.

Thus, we have proved the following theorem.

Theorem 9. Let $l: \mathbb{R}^{n} \rightarrow \mathbb{R}$ be a continuous function and Hamiltonian $H$ satisfies hypotheses $(\mathbf{H 1})-(\mathbf{H} 4)$. Then there exists exactly one minimax solution of the dual problem (in the sense of Definition $3^{\prime}$ ) i.e. a pair of functions $(W, X): \bar{G} \rightarrow \mathbb{R}^{n+1}$ (together with $V(t, p)=W(t, p) y^{0}+X(t, p) y$ ) such that for any $F_{g} \in \mathbf{F}_{g}(H)$ and $F_{d} \in \mathbf{F}_{d}(H)$ the following equality

$$
\operatorname{Sol}_{g}\left(F_{g}\right) \cap \operatorname{Sol}_{d}\left(F_{d}\right)=\{(W, X)\}
$$

holds.

\section{Minimax solutions for nonhomogeneous Hamiltonians}

The dual problem (9), (8), (10) of Hamilton-Jacobi equation we shall call nonhomogenous if the hypothesis $(\mathbf{H} 4)$ is not satisfied. In this section we show that the definition as well the proof of the existence and uniqueness of minimax solution for homogenous Hamiltonian can be translated into the nonhomogenous Hamiltonian.

\subsection{Relations between nonhomogenous and homogenous Hamil- tonians.}

Let us consider the dual problem for a nonhomogenous Hamiltonian

$$
V_{t}(t, p)+H\left(t,-V_{y}(t, p), p\right)=0,(t, p) \in(0, T) \times \mathbb{R}^{n+1},
$$

but we require that the function $V$ satisfies transversality condition

$$
V(t, p)=V_{y^{0}}(t, p) y^{0}+V_{y}(t, p) y,(t, p) \in(0, T) \times \mathbb{R}^{-} \times \mathbb{R}^{n}
$$

and a special type of the boundary condition

$$
y^{0} V_{y^{0}}(T, p)=y^{0} l\left(-V_{y}(T, p)\right) .
$$

For that problem we can formulate the corresponding to it a dual problem for homogenous Hamiltonian

$$
\begin{aligned}
& \bar{V}_{t}(t, p, z)+\bar{H}\left(t,-\bar{V}_{y}(t, p), p, \partial \bar{V}(t, p, z) / \partial z\right)=0, \\
& (t, p) \in(0, T) \times \mathbb{R}^{n+1}, z \in \mathbb{R} \\
& \bar{V}(t, p, z)=\bar{V}_{y^{0}}(t, p, z) y^{0}+\bar{V}_{y}(t, p) y \\
& (t, p, z) \in(0, T) \times \mathbb{R}^{n+1} \times \mathbb{R}
\end{aligned}
$$




$$
y^{0} \bar{V}_{y^{0}}(T, p, z)=y^{0} l\left(-\bar{V}_{y}(T, p)\right)+z .
$$

The solutions of the problems (54), (55), (56) and (57), (58), (59) are related by the equalities $y^{0} \bar{V}_{y^{0}}(t, p, z)=y^{0} V_{y^{0}}(t, p)+z, \bar{V}_{y}(t, p)=V_{y}(t, p),(t, p, z) \in$ $[0, T] \times \mathbb{R}^{n+1} \times \mathbb{R}$. For each $t \in[0, T], x \in \mathbb{R}^{n}, p \in \mathbb{R}^{n+1}, b \in \mathbb{R}$, the Hamiltonian in the problem (57), (58), (59) is defined as follows

$$
\bar{H}(t, x, p, b)= \begin{cases}|b| H(t, x / b, p) & \text { if } b \neq 0, \\ \lim _{b \rightarrow 0} b H(t, x / b, p) & \text { if } b=0 .\end{cases}
$$

We assume that there exists a limit in the right hand side of (60). In the problem (57), (58), (59) a new variable $z$ is introduced however the Hamiltonian (60) does not depend upon that variable. The definition of $\bar{H}$ implies that it is positively homogeneous in $\bar{x}=(x, b)$, i.e.

$$
\begin{aligned}
\bar{H}(t, a x, p, a b)= & a \bar{H}(t, x, p, b) \text { for } a>0, \\
& t \in[0, T], p \in \mathbb{R}^{n+1},(x, b) \in \mathbb{R}^{n+1} .
\end{aligned}
$$

Simultaneously we have

$$
\bar{H}(t, x, p, 1)=H(t, x, p)
$$

for the same $t, x, p$.

Let $V(t, p)$ be a solution of the problem (54), (55), (56). Then from (62) we infer that $y^{0} \bar{V}_{y^{0}}(t, p, z)=y^{0} V_{y^{0}}(t, p)+z, \bar{V}_{y}(t, p)=V_{y}(t, p)$ together with $\bar{V}(t, p, z)$ defined by (58) is a solution of the problem (57), (58), (59). On the other hand, let the function of the form $y^{0} \bar{V}_{y^{0}}(t, p, z)=y^{0} V_{y^{0}}(t, p)+z$, $\bar{V}_{y}(t, p)=V_{y}(t, p)$ together with $\bar{V}(t, p, z)$ defined by (58) be a solution of the problem (57), (58), (59). Then the function $V(t, p)$ defined by (55) is a solution of the problem (54), (55), (56). The latter means that the solutions of the homogeneous and the nonhomogenous dual problem are connected with the relation described above. Of course the relation described is true for the classical solutions only, however it provides, a possibility of such relation. In the next two sections we show that it holds for minimax solutions too.

\subsection{The assumptions.}

Now we formulate the hypotheses which allow us to prove the existence and uniqueness theorem for minimax solutions to nonhomogenous Hamilton-Jacobi problem. Of course, we assume that $l: \mathbb{R}^{n} \rightarrow \mathbb{R}$ is currently continuous too. The hypotheses imposed below on the Hamiltonian are of such a type such that in auxiliary problem with homogeneous Hamiltonian $\bar{H}$ the hypotheses $(\mathbf{H 1})-(\mathbf{H} 4)$ were satisfied.

Therefore we assume on $H$ :

H1' For each $x \in \mathbb{R}^{n}$ the function $(t, p) \rightarrow H(t, x, p)$ is continuous in $G$. 
H2' For each $(t, p) \in[0, T] \times \mathbb{R}^{n+1}$ and $x \in S=\left\{x \in \mathbb{R}^{n}:|x|=1\right\}$ there exists a limit

$$
\lim _{b \downarrow 0} b H(t, x / b, p)=H_{0}(t, x, p),
$$

and for any $x \in S$ the function $(t, p) \rightarrow H_{0}(t, x, p)$ is continuous on $[0, T] \times \mathbb{R}^{n+1}$.

H3' For any bounded set $D \subset[0, T] \times \mathbb{R}^{n+1}$ there exists a number $\Lambda$ such that for all $\left(t, p^{\prime}\right) \in D,\left(t, p^{\prime \prime}\right) \in D,(x, b) \in S_{+}=\{(x, b) \in$ $\left.\mathbb{R}^{n} \times(0, \infty):|x|^{2}+b^{2}=1\right\}$ the following estimation

$$
b\left|H\left(t, x / b, p^{\prime}\right)-H\left(t, x / b, p^{\prime \prime}\right)\right| \leq \Lambda\left|p^{\prime}-p^{\prime \prime}\right|
$$

holds.

H4' For each $(t, p) \in[0, T] \times \mathbb{R}^{n+1},\left(x^{\prime}, b^{\prime}\right) \in B_{+}=\left\{(x, b) \in \mathbb{R}^{n} \times\right.$ $\left.(0, \infty):|x|^{2}+b^{2} \leq 1\right\},\left(x^{\prime \prime}, b^{\prime \prime}\right) \in B_{+}$the following estimation

$\left|b^{\prime} H\left(t, x^{\prime} / b^{\prime}, p\right)-b^{\prime \prime} H\left(t, x^{\prime \prime} / b^{\prime \prime}, p\right)\right| \leq K(t, p)\left(\left|x^{\prime}-x^{\prime \prime}\right|^{2}+\left(b^{\prime}-b^{\prime \prime}\right)^{2}\right)^{1 / 2}$.

holds, where $(t, p) \rightarrow K(t, p)$ is continuous on $[0, T] \times \mathbb{R}^{n+1}$, and there exists a $k>0$, such that

$$
K(t, p) \leq k(1+|p|), \quad(t, p) \in[0, T] \times \mathbb{R}^{n+1} .
$$

If we take $\bar{H}$ defined by (60) and satisfying $\left(\mathbf{H 1}{ }^{\prime}\right)-\left(\mathbf{H} \mathbf{4}^{\prime}\right)$, then it has the following properties:

$\overline{\mathbf{H}} 1$ For each $(x, b) \in \bar{S}=\left\{(x, b) \in \mathbb{R}^{n} \times \mathbb{R}:|x|^{2}+b^{2}=1\right\}$ the function $(t, p) \rightarrow \bar{H}(t, x, p, b)$ is continuous on $[0, T] \times \mathbb{R}^{n+1}$.

$\overline{\mathbf{H}} 2$ For any bounded set $D \subset[0, T] \times \mathbb{R}^{n+1}$ there exists a number $\Lambda$ such that for all $\left(t, p^{\prime}\right) \in D,\left(t, p^{\prime \prime}\right) \in D,(x, b) \in \bar{S}$ the following estimation

$$
\left|\bar{H}\left(t, x, p^{\prime}, b\right)-\bar{H}\left(t, x, p^{\prime \prime}, b\right)\right| \leq \Lambda\left|p^{\prime}-p^{\prime \prime}\right|
$$

holds.

$\overline{\mathbf{H}} 3$ For each $(t, p) \in[0, T] \times \mathbb{R}^{n+1},\left(x^{\prime}, b^{\prime}\right) \in \bar{B}=\left\{(x, b) \in \mathbb{R}^{n} \times \mathbb{R}:|x|^{2}+\right.$ $\left.b^{2} \leq 1\right\},\left(x^{\prime \prime}, b^{\prime \prime}\right) \in \bar{B}$ the following inequality

$$
\left|\bar{H}\left(t, x^{\prime}, p, b^{\prime}\right)-\bar{H}\left(t, x^{\prime \prime}, p, b^{\prime \prime}\right)\right| \leq K(t, p)\left(\left|x^{\prime}-x^{\prime \prime}\right|^{2}+\left(b^{\prime}-b^{\prime \prime}\right)^{2}\right)^{1 / 2}
$$

holds, where the function $(t, p) \rightarrow K(t, p)$ is continuous on $[0, T] \times$ $\mathbb{R}^{n+1}$, and there exists a $k>0$, such that the estimation

$$
K(t, p) \leq k(1+|p|), \quad(t, p) \in[0, T] \times \mathbb{R}^{n+1}
$$

holds.

$\overline{\mathbf{H}} 4$ For each $(t, p) \in[0, T] \times \mathbb{R}^{n+1}$ the function $(x, b) \rightarrow \bar{H}(t, x, p, b)$ is positively homogeneous, i.e. the condition (61) is satisfied. 


\subsection{Upper, lower and minimax solutions of nonhomogenous dual problem.}

Let us consider the dual problem (57), (58), (59) with Hamiltonian $\bar{H}$ satisfying $(\overline{\mathbf{H}} \mathbf{1})-(\overline{\mathbf{H}} \mathbf{4})$. If we compare $(\overline{\mathbf{H}} \mathbf{1})-(\overline{\mathbf{H}} \mathbf{4})$ with $(\mathbf{H} \mathbf{1})-(\mathbf{H} \mathbf{4})$ we easily see that in the new case we can apply the Theorem 9 in view of which there exists exactly one minimax solution of the problem (57), (58), (59), i.e. we have the following theorem.

Theorem 10. Let Hamiltonian H satisfy hypotheses (H1')-(H4') or Hamiltonian $\bar{H}$ satisfies $(\overline{\mathbf{H}} \mathbf{1})-(\overline{\mathbf{H}} \mathbf{4})$, and let $l: \mathbb{R}^{n} \rightarrow \mathbb{R}$ be continuous. Then there exists exactly one pair of functions $(\bar{W}, \bar{X}): \bar{G} \rightarrow \mathbb{R}^{n+1}$ being (together with $\left.\bar{V}(t, p, z)=\bar{W}(t, p, z) y^{0}+\bar{X}(t, p) y\right)$ a minimax solution of the dual problem (57), (58), (59). Furthermore for this solution we have the relations

$$
\bar{V}(t, p, z)=\bar{V}(t, p, 0)+z, \quad(t, p) \in[0, T] \times \mathbb{R}^{n+1}, z \in \mathbb{R} .
$$

The proof of the theorem is based on the Lemma 11, proved below. We will need the following multivalued mapping:

$$
(t, p, q) \rightarrow \bar{F}_{g}(t, q, p) \subset \mathbb{R}^{n+1} \times \mathbb{R},(t, p, r) \rightarrow \bar{F}_{d}(t, r, p) \subset \mathbb{R}^{n+1} \times \mathbb{R},
$$

where $(t, p) \in[0, T] \times \mathbb{R}^{n+1}, q \in Q, r \in P$.

Define them as follows:

$$
\begin{aligned}
\bar{F}(t, p)= & \left\{(f, g) \in \mathbb{R}^{n+1} \times R: f=(0, \dot{y}),|f|^{2}+g^{2} \leq 2 K^{2}(t, p)\right\}, \\
\bar{F}_{g}(t, x, p, b)= & \{(f, g) \in \bar{F}(t, p): \\
& f=(0, \dot{y}),\langle-\dot{y}, x\rangle+b g \geq \bar{H}(t, x, p, b)\}, \\
\bar{F}_{d}(t, x, p, b)= & \{(f, g) \in \bar{F}(t, p): \\
& f=(0, \dot{y}),\langle-\dot{y}, x\rangle+b g \leq \bar{H}(t, x, p, b)\},
\end{aligned}
$$

where $(t, p) \in[0, T] \times \mathbb{R}^{n+1},(x, b)=q=r \in \mathbb{R}^{n} \times \mathbb{R}=Q=P$, and $K(t, p)$ is the same as in $\left(\mathbf{H} \mathbf{4}^{\prime}\right)$. We easily see that $\bar{F}_{g}$ and $\bar{F}_{d}$ satisfy the conditions:

1. For each $(t, p, q, r) \in G \times Q \times P$ the sets $\bar{F}_{g}(t, q, p)$ and $\bar{F}_{d}(t, r, p)$ are nonempty, convex and compact in $\mathbb{R}^{n+1} \times \mathbb{R}$; for each $t \in(0, T)$, $r \in P, q \in Q$ and $(f, g) \in \bar{F}_{g}(t, q, p) \cup \bar{F}_{d}(t, r, p)$ we have the inequality

$$
\left(|f|^{2}+g^{2}\right)^{1 / 2} \leq(1+|p|) c, c>0 \text { - some constant. }
$$

2. For each $(q, r) \in Q \times P$ the multivalued mappings $(t, p) \rightarrow \bar{F}_{g}(t, q, p)$, $(t, p) \rightarrow \bar{F}_{d}(t, r, p)$ are upper semicontinuous. 
3. For any $(t, p) \in G$ and $(x, b) \in \mathbb{R}^{n} \times \mathbb{R}$ we have equalities

$$
\begin{aligned}
& \sup _{q \in Q} \min _{(0, \dot{y}, g) \in \bar{F}_{g}(t, q, p)}\{\langle-\dot{y}, x\rangle+b g\}=\bar{H}(t, x, p, b), \\
& \inf _{r \in P} \max _{(0, \dot{y}, g) \in \bar{F}_{d}(t, r, p)}\{\langle-\dot{y}, x\rangle+b g\}=\bar{H}(t, x, p, b) .
\end{aligned}
$$

A set of all mappings $\bar{F}_{g}$ and $\bar{F}_{d}$ satisfying conditions 1.-3. will be denoted by $\mathbf{F}_{g}(\bar{H}), \mathbf{F}_{d}(\bar{H})$, respectively. Of course $\mathbf{F}_{g}(\bar{H}) \neq \emptyset$ and $\mathbf{F}_{d}(\bar{H}) \neq \emptyset$.

Take any $\bar{F}_{g} \in \mathbf{F}_{g}(\bar{H})$ and $\bar{F}_{d} \in \mathbf{F}_{d}(\bar{H})$. Let us consider differential inclusions

$$
\begin{gathered}
d \bar{p}(t) / d t \in \bar{F}_{g}(t, q, p(t)), \\
d \bar{p}(t) / d t \in \bar{F}_{d}(t, r, p(t)), \\
|d \bar{p}(t) / d t| \leq(1+|p(t)|) c .
\end{gathered}
$$

Here $\bar{p}(t)=(p(t), z(t)) \in \mathbb{R}^{n+1} \times \mathbb{R}, t \in\left[t_{0}, T\right],|\bar{p}(t)|=\left(|p(t)|^{2}+z^{2}(t)\right)^{1 / 2}$. The set of all solutions of (69) (respectively (70) and (71)), satisfying the initial condition $\bar{p}\left(t_{0}\right)=\left(p_{0}, z_{0}\right)$, will be denoted by $\bar{Y}_{g}\left(t_{0}, p_{0}, z_{0}, q\right)$ (respectively $\bar{Y}_{d}\left(t_{0}, p_{0}, z_{0}, r\right)$ and $\left.\bar{Y}\left(t_{0}, p_{0}, z_{0}\right)\right)$.

By $\operatorname{Sol}_{g}^{0}\left(\bar{F}_{g}\right)$ denote the set of all pairs of functions $\left(\bar{W}_{g}, \bar{X}_{g}\right):[0, T] \times$ $\mathbb{R}^{n+1} \times \mathbb{R} \rightarrow \mathbb{R} \times \mathbb{R}^{n}$, which satisfy the inequalities

$$
\begin{aligned}
& \bar{W}_{g}(T, p, z) y^{0} \geq y^{0} l(-\bar{X}(T, p))+z, \\
& \sup _{\tau \in\left(t_{0}, T\right], q \in Q} \quad \inf _{\bar{p}(\cdot) \in \bar{Y}_{g}\left(t_{0}, p_{0}, z_{0}, q\right)} \bar{V}_{g}(\tau, p(\tau), z(\tau)) \leq \bar{V}_{g}\left(t_{0}, p_{0}, z_{0}\right),
\end{aligned}
$$

where $\bar{V}_{g}(t, p, z)=\bar{W}_{g}(t, p, z) y^{0}+\bar{X}_{g}(t, p) y, t_{0} \in[0, T), p_{0} \in \mathbb{R}^{n+1}$ and $\bar{V}_{g}(\cdot, \cdot, \cdot)$ is lower semicontinuous and $\bar{X}_{g}(T, \cdot)$ is continuous. Analogously we define $\operatorname{Sol}_{d}^{0}\left(\bar{F}_{d}\right)$. According to the theorem 9 there exists exactly one pair of functions (a minimax solution of dual problem (57), (58), (59)) $(\bar{W}, \bar{X}):[0, T] \times \mathbb{R}^{n+1} \times \mathbb{R} \rightarrow \mathbb{R} \times \mathbb{R}^{n}$ such that for any $\bar{F}_{g} \in \mathbf{F}_{g}(\bar{H})$ and $\bar{F}_{d} \in \mathbf{F}_{d}(\bar{H})$ we have equality

$$
\operatorname{Sol}_{g}^{0}\left(\bar{F}_{g}\right) \cap \operatorname{Sol}_{d}^{0}\left(\bar{F}_{d}\right)=\{(\bar{W}, \bar{X})\} .
$$

Lemma 11. Let $\bar{F}_{g} \in \mathbf{F}_{g}(\bar{H})$ and $\bar{F}_{d} \in \mathbf{F}_{d}(\bar{H})$. For each pair $\left(\bar{W}_{g}, \bar{X}\right) \in$ $\operatorname{Sol}_{g}^{0}\left(\bar{F}_{g}\right)$ and $\left(\bar{W}_{d}, \bar{X}\right) \in \operatorname{Sol}_{d}^{0}\left(\bar{F}_{d}\right)$ (with the same $\bar{X}$ ) we have, for $\bar{V}_{g}(t, p, z)=\bar{W}_{g}(t, p, z) y^{0}+\bar{X}(t, p) y$ and $\bar{V}_{d}(t, p, z)=\bar{W}_{d}(t, p, z) y^{0}+\bar{X}(t, p) y$, the inequalities

$$
\begin{aligned}
& \bar{V}_{g}(t, p, 0)+z \geq \bar{V}_{d}(t, p, z),(t, p) \in \bar{G} \text { and } z \in \mathbb{R} \\
& \bar{V}_{d}(t, p, 0)+z \leq \bar{V}_{g}(t, p, z),(t, p) \in \bar{G} \text { and } z \in \mathbb{R} .
\end{aligned}
$$


Proof. We prove the inequality (73). To this effect we use the constructions introduced in the proof of the lemma 8. Let us consider the set

$$
\begin{aligned}
W_{a}= & \left\{\left(\bar{p}_{g}(\cdot), \bar{p}_{d}(\cdot)\right) \in \bar{Y}\left(t_{0}, p_{0}, 0\right) \times \bar{Y}\left(t_{0}, p_{0}, z_{0}\right):\right. \\
& \left.\langle\bar{s}(t), d \bar{s}(t) / d t\rangle \leq \Lambda|\bar{s}(t)|^{2}+a \text { for a.e. } t \in\left[t_{0}, T\right]\right\},
\end{aligned}
$$

where $\bar{p}_{g}(t)=\left(p_{g}(t), z_{g}(t)\right), \bar{p}_{d}(t)=\left(p_{d}(t), z_{d}(t)\right), \bar{s}(t)=\left(y_{d}(t)-y_{g}(t)\right.$, $\left.z_{d}(t)-z_{g}(t)-z_{0}\right)$. Notice that $\bar{s}\left(t_{0}\right)=(0,0) \in \mathbb{R}^{n} \times \mathbb{R}$. Set

$$
\begin{aligned}
M_{a}(t)= & \left\{\left(\bar{p}_{g}(\cdot), \bar{p}_{d}(\cdot)\right) \in W_{a}:\right. \\
& \bar{V}_{g}\left(t_{0}, p_{0}, 0\right) \geq \bar{V}_{g}\left(t, p_{g}(t), z_{g}(t)\right), \\
& \left.\bar{V}_{d}\left(t_{0}, p_{0}, z_{0}\right) \leq \bar{V}_{d}\left(t, p_{d}(t), z_{d}(t)\right)\right\} \\
t_{a}= & \sup \left\{t \in\left[t_{0}, T\right]: M_{a}(t) \neq \emptyset\right\} .
\end{aligned}
$$

Analogously as in Lemma 8 we prove that $t_{a}=T$. On the contrary, let us assume $t_{a}<T$. Let $t^{0}=t_{a},\left(\bar{p}_{g}^{0}(\cdot), \bar{p}_{d}^{0}(\cdot)\right) \in M_{a}\left(t^{0}\right)$. Put $\left(y_{0}^{0}, y_{g 0}, z_{g}^{0}\right)=$ $\left(p_{g}^{0}, z_{g}^{0}\right)=\bar{p}_{g}^{0}\left(t^{0}\right),\left(y_{0}^{0}, y_{d 0}, z_{d}^{0}\right)=\left(p_{d}^{0}, z_{d}^{0}\right)=\bar{p}_{d}^{0}\left(t^{0}\right), \bar{s}^{0}=\left(s^{0}, b^{0}\right)=\left(y_{d 0}, z_{d}^{0}\right)-$ $\left(y_{g 0}, z_{g}^{0}\right)$. From $(76)$ we obtain the inequalities

$$
\bar{V}_{g}\left(t_{0}, p_{0}, 0\right) \geq \bar{V}_{g}\left(t^{0}, p_{g}^{0}, z_{g}^{0}\right), \quad \bar{V}_{d}\left(t_{0}, p_{0}, z_{0}\right) \leq \bar{V}_{d}\left(t^{0}, p_{d}^{0}, z_{d}^{0}\right) .
$$

Taking into account Property 3 . let us choose $r_{a} \in P$, and $q_{a} \in Q$ such that (we have in mind that $f_{g}=\left(0, \dot{y}_{g}\right)$ and $f_{d}=\left(0, \dot{y}_{d}\right)$ )

$$
\begin{aligned}
& \left\langle s^{0}, \dot{y}_{g}\right\rangle+b^{0} g_{g} \geq \bar{H}\left(t^{0},-s^{0}, p_{g}^{0}, b^{0}\right)-a / 4, \quad\left(f_{g}, g_{g}\right) \in \bar{F}_{g}\left(t^{0}, q_{a}, p_{g}^{0}\right), \\
& \left\langle s^{0}, \dot{y}_{d}\right\rangle+b^{0} g_{d} \leq \bar{H}\left(t^{0},-s^{0}, p_{d}^{0}, b^{0}\right)+a / 4, \quad\left(f_{d}, g_{d}\right) \in \bar{F}_{d}\left(t^{0}, r_{a}, p_{d}^{0}\right) .
\end{aligned}
$$

Since the mappings $(t, p) \rightarrow \bar{F}_{g}\left(t, q_{a}, p\right),(t, p) \rightarrow \bar{F}_{d}\left(t, r_{a}, p\right)$ are upper semicontinuous, and Hamiltonian $\bar{H}$ is continuous with respect to its all variables, there exists a $\delta>0$ such that for almost all $t \in\left[t^{0}, t^{0}+\delta\right]$ and for all $\left(y_{0}^{0}, y_{g}(\cdot), z_{g}(\cdot)\right)=\bar{p}_{g}(\cdot) \in \bar{Y}_{g}\left(t^{0}, p_{g}^{0}, q_{a}, z_{g}^{0}\right),\left(y_{0}^{0}, y_{d}(\cdot), z_{d}(\cdot)\right)=\bar{p}_{d}(\cdot) \in$ $\bar{Y}_{d}\left(t^{0}, p_{d}^{0}, r_{a}, z_{d}^{0}\right)$ the following inequalities

$$
\begin{aligned}
& \left\langle s(t), \dot{y}_{g}(t)\right\rangle+b(t) \dot{z}_{g}(t) \geq \bar{H}\left(t,-s(t), p_{g}(t), b(t)\right)-a / 2, \\
& \left\langle s(t), \dot{y}_{d}(t)\right\rangle+b(t) \dot{z}_{d}(t) \leq \bar{H}\left(t,-s(t), p_{d}(t), b(t)\right)+a / 2,
\end{aligned}
$$

hold, where $s(t)=y_{d}(t)-y_{g}(t), b(t)=z_{d}(t)-z_{g}(t), p_{g}(t)=\left(y_{0}^{0}, y_{g}(t)\right)$, $p_{d}(t)=\left(y_{0}^{0}, y_{d}(t)\right)$. Notice that $\left(t, p_{d}(t)\right) \in E,\left(t, p_{g}(t)\right) \in E, t^{0} \leq t \leq t^{0}+\delta$. By $(\overline{\mathbf{H}} \mathbf{2})$ and $(\overline{\mathbf{H}} \mathbf{4})$ we further get

$$
\begin{aligned}
& \langle\bar{s}(t), d \bar{s}(t) / d t\rangle=\left\langle s(t), \dot{y}_{d}(t)\right\rangle-\left\langle s(t), \dot{y}_{g}(t)\right\rangle+b(t)\left(\dot{z}_{d}(t)-\dot{z}_{g}(t)\right) \\
& \leq \bar{H}\left(t,-s(t), p_{d}(t), b(t)\right)-\bar{H}\left(t,-s(t), p_{g}(t), b(t)\right)+a \\
& \leq \Lambda|\bar{s}(t)||s(t)|+a \leq \Lambda|\bar{s}(t)|^{2}+a,
\end{aligned}
$$

where $\bar{s}(t)=(s(t), b(t))$. 
Next similarly as in the proof of the Lemma 8 define the pair $\left(\bar{p}_{g}{ }_{g}(\cdot)\right.$, $\left.\bar{p}_{d}{ }_{d}(\cdot)\right) \in M_{a}\left(t^{0}+\delta\right)$. Then we come to a contradiction with the definition of the $t_{a}$, hence $t_{a}=T$.

By the definition of $M_{a}(t)$ and the equality $t_{a}=T$ we have

$$
\begin{aligned}
\bar{V}_{g}\left(t_{0}, p_{0}, 0\right) & \geq \bar{V}_{g}\left(T, p_{g}^{k}(T), z_{g}^{k}(T)\right) \\
& \geq \bar{X}\left(T, p_{g}^{k}\right) y_{g}^{k}+y_{g}^{k 0} l\left(-\bar{X}\left(T, p_{g}^{k}(T)\right)+z_{g}^{k}(T),\right. \\
\bar{V}_{d}\left(t_{0}, p_{0}, z_{0}\right) & \leq \bar{V}_{d}\left(T, p_{d}^{k}(T), z_{d}^{k}(T)\right) \\
& \leq \bar{X}\left(T, p_{d}^{k}\right) y_{d}^{k}+y_{d}^{k 0} l\left(-\bar{X}\left(T, p_{d}^{k}(T)\right)+z_{d}^{k}(T),\right.
\end{aligned}
$$

where $\left(\bar{p}_{g}^{k}(\cdot), \bar{p}_{d}^{k}(\cdot)\right) \in M_{a_{k}}(T), a_{k} \rightarrow 0$, when $k \rightarrow \infty$. We can assume, without loss the generality that $\bar{p}_{g}^{k}(\cdot) \rightarrow \bar{p}_{g}^{*}(\cdot)$ and $\bar{p}_{d}^{k}(\cdot) \rightarrow \bar{p}_{d}^{*}(\cdot)$ when $k \rightarrow$ $\infty$. In turn, by the definition of $W_{a}$, we have

$$
p_{g}^{*}(t)=p_{d}^{*}(t), \quad z_{g}^{*}(t)+z_{0}=z_{d}^{*}(t), \quad t_{0} \leq t \leq T .
$$

Hence, we conclude

$$
\begin{aligned}
\bar{V}_{g}\left(t_{0}, p_{0}, 0\right)+z_{0} & \geq \bar{X}\left(T, p_{g}^{*}\right) y_{g}^{*}+y_{g}^{* 0} l\left(-\bar{X}\left(T, p_{g}^{*}(T)\right)\right)+z_{g}^{*}(T)+z_{0} \\
& =\bar{X}\left(T, p_{d}^{*}\right) y_{d}^{*}+y_{d}^{* 0} l\left(-\bar{X}_{d}\left(T, p_{d}^{*}(T)\right)\right)+z_{d}^{*}(T) \\
& \geq \bar{V}_{d}\left(t_{0}, p_{0}, z_{0}\right) .
\end{aligned}
$$

Therefore the first inequality in the assertion of the lemma is proved. Analogously one can prove the second one in (74). The lemma is proved.

The inequalities (74), (73) and (72) imply the equality (63) and therefore the Theorem 2 is proved.

Let us come back to our problem (9), (8), (10) with the nonhomogeneous Hamiltonian $H$. We shall call a minimax solution of that problem a pair of functions $(W, X)$ such that for $V(t, p)=W(t, p) y^{0}+X(t, p) y$

$$
V(t, p)=\bar{V}(t, p, 0), X(t, p)=\bar{X}(t, p),(t, p) \in[0, T] \times \mathbb{R}^{n+1},
$$

where $(\bar{W}, \bar{X})\left(\bar{V}(t, p, z)=\bar{W}(t, p, z) y^{0}+\bar{X}(t, p) y\right)$ is the minimax solution of the dual problem (57), (58), (59).

Define now an upper and a lower solution of the problem (9), (8), (10) with the nonhomogeneous Hamiltonian.

Definition 4. Let $\bar{F}_{g} \in \mathbf{F}_{g}(\bar{H})$. By $\operatorname{Sol}_{g}\left(\bar{F}_{g}\right)$ denote the set of all pairs of functions $W(t, p), \quad X(t, p), \quad(t, p) \in \bar{G}$, such that $V(\cdot, \cdot)(V(t, p)=$ $\left.W(t, p) y^{0}+X(t, p) y\right)$ is lower semicontinuous and $X(T, \cdot)$ is continuous, $V(T, p) \geq X(T, p) y+y^{0} l(-X(T, p)), p \in \mathbb{R}^{n+1}$ and the following condition

$$
\sup _{\tau \in\left(t_{0}, T\right], q \in \mathbb{R}^{n}} \quad \inf _{\bar{p}(\cdot) \in \bar{Y}_{g}\left(t_{0}, p_{0}, 0, q\right)}[V(\tau, p(\tau))+z(\tau)] \leq V\left(t_{0}, p_{0}\right),
$$


is satisfied for $\left(t_{0}, p_{0}\right) \in[0, T] \times \mathbb{R}^{n+1}$. An upper solution of (9), (8), (10) we shall call each pair of functions $(W, X) \in \operatorname{Sol}_{g}\left(\bar{F}_{g}\right)$.

Definition 5. Let $\bar{F}_{d} \in \mathbf{F}_{d}(\bar{H})$. By $\operatorname{Sol}_{d}\left(\bar{F}_{d}\right)$ denote the set of all pairs of functions $W(t, p), \quad X(t, p), \quad(t, p) \in \bar{G}$, such that $V(\cdot, \cdot)(V(t, p)=$ $\left.W(t, p) y^{0}+X(t, p) y\right)$ is upper semicontinuous and $X(T, \cdot)$ is continuous, $V(T, p) \leq X(T, p) y+y^{0} l(-X(T, p)), p \in \mathbb{R}^{n+1}$ and the following condition

$$
\inf _{\tau \in\left(t_{0}, T\right], r \in \mathbb{R}^{n}} \sup _{\bar{p}(\cdot) \in \bar{Y}_{d}\left(t_{0}, p_{0}, 0, r\right)}[V(\tau, p(\tau))+z(\tau)] \geq V\left(t_{0}, p_{0}\right),
$$

is satisfied for $\left(t_{0}, p_{0}\right) \in[0, T] \times \mathbb{R}^{n+1}$. A lower solution of (9), (8), (10) we shall call each pair of functions $(V, X) \in \operatorname{Sol}_{d}\left(\bar{F}_{d}\right)$.

Definition 6. A minimax solution to the problem (9), (8), (10) (with nonhomogeneous Hamiltonian) we shall call a pair of functions $W(t, p), X(t, p)$, $(t, p) \in \bar{G}$, such that $V(\cdot, \cdot)\left(V(t, p)=W(t, p) y^{0}+X(t, p) y\right)$ and $X(T, \cdot)$ are continuous and which are simultanously an upper and lower solution to that problem, i.e. $(W, X) \in \operatorname{Sol}_{g}\left(\bar{F}_{g}\right) \cap \operatorname{Sol}_{d}\left(\bar{F}_{d}\right)$.

As a consequence of the relations between the minimax solutions for nonhomogeneous and homogeneous our dual problem from Theorem 9 follows the main theorem of the paper.

Theorem 12. Let $l: \mathbb{R}^{n} \rightarrow \mathbb{R}$ be a continuous and Hamiltonian $H$ satisfies $\left(\mathbf{H 1}{ }^{\prime}\right)-\left(\mathbf{H} 4^{\prime}\right)$. Then there exists exactly one pair of functions $(W, X): \bar{G} \rightarrow$ $\mathbb{R}^{n+1}$ (together $V(t, p)=W(t, p) y^{0}+X(t, p) y$ a minimax solution to dual problem with nonhomogeneous Hamiltonian) such that for any $\bar{F}_{g} \in \mathbf{F}_{g}(\bar{H})$ and $\bar{F}_{d} \in \mathbf{F}_{d}(\bar{H})$ we have the equality

$$
\operatorname{Sol}_{g}\left(\bar{F}_{g}\right) \cap \operatorname{Sol}_{d}\left(\bar{F}_{d}\right)=\{(W, X)\} .
$$

\section{Example}

Let us consider a function $\hat{H}: \mathbb{R} \rightarrow \mathbb{R}$ which satisfies the hypotheses (H3) and $(\mathbf{H} 4)$ with respect to $x$. Define the Hamiltoniam

$$
H(t, x, p)=-\frac{1}{2} \frac{\left(y^{0}\right)^{2}}{y} \hat{H}(x)
$$

in a set $[0, T] \times \mathbb{R} \times P$, where $P=\left\{\left(y^{0}, y\right) \in \mathbb{R}^{2}: y^{0} \leq 0, y>0\right\}$. Then our dual Hamilton-Jacobi equation has the form

$$
V_{t}\left(t, \frac{1}{2} p\right)-\frac{1}{2} \frac{\left(y^{0}\right)^{2}}{y} \hat{H}\left(-V_{y}\left(t, \frac{1}{2} p\right)\right)=0,(t, p) \in(0, T) \times P .
$$


We assume that $V$ satisfies the boundary condition: $y^{0} V_{y^{0}}(T, p / 2)$ $=y^{0} T \hat{H}(-a / 2)$ for some constant $a$ and all $p \in P$. We easily check that the function

$$
V\left(t, \frac{1}{2} p\right)=\frac{1}{4} a y^{2}+\frac{1}{4}\left(y^{0}\right)^{2} t \hat{H}\left(-\frac{1}{2} a\right)
$$

satisfies $\left(^{*}\right)$, the boundary condition: $y^{0} V_{y^{0}}(T, p / 2)=y^{0} T \hat{H}(-a / 2)$ and (8). We see that $\left({ }^{*}\right)$ is a classical solution of $\left(^{*}\right)$ and so by Section 2.1 it is also a generalized solution of $(*)$. Therefore we obtained that some class of nonlinear equations have a solution given by formula $(* *)$.

\section{Dependence on parameters}

In the last section we shall consider a problem of dependence on parameters and the correctness of minimax solutions.

To this effect let $l_{k}: \mathbb{R}^{n} \rightarrow \mathbb{R}, k=1,2, \ldots$, be a sequence of continuous functions which is uniformly convergent to $l_{*}: \mathbb{R}^{n} \rightarrow \mathbb{R}$, on any compact set $M \subset \mathbb{R}^{n}$. Let $H_{k}: G \times \mathbb{R}^{n} \rightarrow \mathbb{R}, k=1,2, \ldots$, be a sequence of Hamiltonians satisfying $\left(\mathbf{H} \mathbf{1}^{\prime}\right)-\left(\mathbf{H} \mathbf{4}^{\prime}\right)$. We assume that the sequence of Hamiltonians $H_{k}$ is convergent (when $k \rightarrow \infty$ ) to some Hamiltonian $H_{*}$ in the following sense: for each $x \in \mathbb{R}^{n}$ the sequence $H_{k}(\cdot, x, \cdot): G \rightarrow \mathbb{R}$ and $K_{k}: G \rightarrow \mathbb{R}$, $k=1,2, \ldots$, is convergent uniformly on any compact $D \subset \mathbb{R}^{n}$ to functions $H_{*}(\cdot, x, \cdot), K_{*}$ respectively, where $K_{k}, K_{*}$ are functions related to $H_{k}, H_{*}$, as in $\left(\mathbf{H} 4^{\prime}\right)$.

Theorem 13. Let $l_{k}$ and $H_{k}, k=1,2, \ldots$, be the above described sequences of functions convergent to $l_{*}, H_{*}$, respectively. Let $\left(W_{k}, X_{k}\right): \bar{G} \rightarrow \mathbb{R}^{n+1}$, $k=1,2, \ldots$, be a sequence of minimax solutions of the dual problems

$$
\begin{gathered}
V_{t}(t, p)+H_{k}\left(t,-V_{y}(t, p), p\right)=0, \quad(t, p) \in(0, T) \times \mathbb{R}^{-} \times \mathbb{R}^{n}, \\
V(t, p)=V_{y^{0}}(t, p) y^{0}+V_{y}(t, p) y,(t, p) \in(0, T) \times \mathbb{R}^{-} \times \mathbb{R}^{n}, \\
y^{0} V_{y^{0}}(T, p)=y^{0} l_{k}\left(-V_{y}(T, p)\right),
\end{gathered}
$$

such that the sequence $\left\{X_{k}(T, \cdot)\right\}$ is uniformly convergent on any compact set. Then the sequences $V_{k}, X_{k}(T, \cdot), k=1,2, \ldots,\left(V_{k}(t, p)=W_{k}(t, p) y^{0}+\right.$ $\left.X_{k}(t, p) y\right)$ are convergent uniformly, on any compact set $D \subset \bar{G}$, to limit functions $V_{*}, X_{*},\left(X_{k}(\cdot, \cdot) \rightarrow X_{*}(\cdot, \cdot)\right.$ pointwise $)$. The limit pair of functions $W_{*}, X_{*}\left(W_{*}(t, p) y^{0}=V_{*}(t, p)-X_{*}(t, p) y\right)$ is a minimax solution to the problem

$$
\begin{gathered}
V_{t}(t, p)+H_{*}\left(t,-V_{y}(t, p), p\right)=0, \quad(t, p) \in(0, T) \times \mathbb{R}^{-} \times \mathbb{R}^{n}, \\
V(t, p)=V_{y^{0}}(t, p) y^{0}+V_{y}(t, p) y, \quad(t, p) \in(0, T) \times \mathbb{R}^{-} \times \mathbb{R}^{n}, \\
y^{0} V_{y^{0}}(T, p)=y^{0} l_{*}\left(-V_{y}(T, p)\right) .
\end{gathered}
$$


Proof. By our assumptions the functions $l_{k}$ and $X_{k}(T, \cdot)$ are uniformly bounded on any bounded set $C \subset \mathbb{R}^{n}$. This is why (we argue analogously as in the proof of Lemma 1) the functions $V_{k}$ are uniformly bounded below on any bonded set $D \subset \bar{G}$, too.

For $(t, p) \in \bar{G}, \varepsilon>0, K$-natural number, let us put

$$
\begin{aligned}
Z_{-}\left(t, p, K, X_{K}, \varepsilon\right)= & \inf \left\{V_{k}(s, z):\right. \\
& V_{k}(T, p) \geq X_{K}(T, p) y+y^{0} l_{K}\left(-X_{K}(T, p)\right), \\
& k \geq K,|t-s|+|p-z| \leq \varepsilon,(s, z) \in \bar{G}\}, \\
V_{-}(t, p)= & \lim _{K \rightarrow \infty, \varepsilon \rightarrow 0} Z_{-}\left(t, p, K, X_{K}, \varepsilon\right) .
\end{aligned}
$$

Since the functions $V_{k}, k=1,2, \ldots$, are uniformly bounded below on bounded set, therefore the function $Z_{-}$is of finite values. Define a function $X_{*}(t, p)$ as any function for which we have equality $X_{*}(t, p) y=\liminf _{k \rightarrow \infty} X_{k}(t, p) y$, $(t, p) \in \bar{G}$. We easily check (repeating the proof of Lemma 6) that $V_{-}$ is lower semicontinuous and that $V_{-}(T, p) \geq X_{*}(T, p) y+y^{0} l_{*}\left(-X_{*}(T, p)\right.$, $(T, p) \in \bar{G}$, and that $V_{-}$satisfies (78). As a consequence of that we obtain that the pair $\left(W_{-}, X_{*}\right)\left(W_{-}(t, p) y^{0}=V_{-}(t, p)-X_{*}(t, p) y\right)$ is an upper solution of the problem (83), (84), (85).

Analogously we define a lower solution to (83), (84), (85): for $(t, p) \in \bar{G}$, $\varepsilon>0, K$-natural number, let us put

$$
\begin{aligned}
Z_{+}\left(t, p, K, X_{K}, \varepsilon\right)= & \sup \left\{V_{k}(s, z):\right. \\
& V_{k}(T, p) \leq X_{K}(T, p) y+y^{0} l_{K}\left(-X_{K}(T, p)\right), \\
& k \geq K,|t-s|+|p-z| \leq \varepsilon,(s, z) \in \bar{G}\}, \\
V_{+}(t, p)= & \lim _{K \rightarrow \infty, \varepsilon \rightarrow 0} Z_{+}\left(t, p, K, X_{K}, \varepsilon\right) .
\end{aligned}
$$

We also check that the pair $\left(W_{+}, X_{*}\right)\left(W_{+}(t, p) y^{0}=V_{+}(t, p)-X_{*}(t, p) y\right)$ is a lower solution to (83), (84), (85).

As any upper and any lower solutions are related by inequality $V_{g} \geq V_{d}$, therefore $V_{-}(t, p) \geq V_{+}(t, p),(t, p) \in \bar{G}$. On the other hand from (86) and (88) we have $V_{-}(t, p) \leq V_{+}(t, p)$. Both inequalities imply

$$
V_{*}(t, p)=V_{-}(t, p)=V_{+}(t, p),(t, p) \in \bar{G} .
$$

Of course the pair $\left(W_{*}, X_{*}\right)\left(W_{*}(t, p) y^{0}=V_{*}(t, p)-X(t, p) y\right)$ is a minimax solution to (83), (84), (85). By the definition of $V_{-}$and $V_{+}$we obtain that the sequence $V_{k}, k=1,2, \ldots$, is convergent and its limit (together with $\left.\left(W_{*}, X_{*}\right)\right)$ is a minimax solution to $(83),(84),(85)$. 


\section{References}

[1] Frankowska, H., Lower semicontinuous solutions of Hamilton-Jacobi-Bellman equation, SIAM J. Control Optim. 31(1993), 257-272.

[2] Frankowska, H., Plaskacz, S., Rzeżuchowski, T., Measurable viability theorems and Hamilton-Jacobi-Bellman equation, J. Differential Equations 116 (1995), 265-305.

[3] Frankowska, H., Plaskacz, S., Semicontinuous solutions of Hamilton-Jacobi-Bellman equations with state constraints, Cahiers Centre Rech. Viab. Jeux Control 9809 (1998).

[4] Lions, P. L., Optimal Control and Viscosity Solutions, Lecture Notes in Math. 1119, Springer, Berlin, 1985.

[5] Lions, P. L., Neuman type boundary conditions for Hamilton-Jacobi equations, Duke Math. J. 52 (1985), 793-820.

[6] Nowakowska, I., Dual Game Theory and Its Applications in Economic, PhD thesis, Univ. Łódź, Department of Statistics, Łódź, 1998.

[7] Nowakowski, A., Field theories in the modern calculus of variations, Trans. Amer. Math. Soc. 309 (1988), 725-752.

[8] Nowakowski, A., The dual dynamic programming, Proc. Amer. Math. Soc. 116 (1992), 1089-1096.

[9] Sobieski, S., Dual Approach to Game Theory, PhD thesis, Univ. Łódź, Faculty of Mathematics, Łódź, 2002.

[10] Subbotin, A. I., Minimax Inequalities and Hamilton-Jacobi Equations, Nauka, Moscow, 1991.

ANDRZEJ NOWAKOWSKI

FACUlTy OF Mathematics

UNIVERSITY OF ŁÓDŹ

BANACHA 22

90-238 ŁÓDŹ, POLAND

E-MAIL: ANNOWAKO@IMUL.UNI.LODZ.PL 